Supporting information for:

\title{
Anions of Tridentate SPS Ligands: Syntheses, X-Ray Structures and DFT Calculations.
}

\author{
Marjolaine Doux, ${ }^{\text {a }}$ Pierre Thuéry, ${ }^{\mathrm{b}}$ Matthias Blug, ${ }^{\text {a }}$ Louis Ricard, ${ }^{\mathrm{a}}$ Pascal Le Floch, \\ Thérèse Arliguie, ${ }^{b *}$ Nicolas Mézailles ${ }^{a} *$ \\ ${ }^{\text {a }}$ Laboratoire « Hétéroéléments et Coordination », Ecole Polytechnique, CNRS, 91128 \\ Palaiseau Cédex, France, Tel: +3316933 4414 ; Fax: +331693344 49 \\ E-mail: nicolas.mezailles@ polytechnique.edu \\ ${ }^{\mathrm{b}}$ Service de Chimie Moléculaire, DSM, DRECAM, CNRS URA 331, CEA/Saclay, 91191 \\ Gif-sur-Yvette cedex, France, Tel: +3316908 6042 ; Fax: +33 169086640 \\ E-mail: therese.arliguie@cea.fr
}




\section{Index}

\section{Spectral-Data}

NMR-Spectra of compound 2

NMR-Spectra of compound $\mathbf{3} \quad 6$

NMR-Spectra of compounds 4 and 5

NMR-Spectrum of compound 7

NMR-Spectra of compound 8a 13

NMR-Spectrum of compounds $\mathbf{8 b}$ and $\mathbf{8 c} \quad 15$

NMR-Spectrum of compound $9 \quad 16$

NMR-Spectra of compound $\mathbf{1 0} \quad 17$

$\begin{array}{ll}\text { NMR-Spectra of compound } \mathbf{1 1} & 18\end{array}$

NMR-Spectra of compound 12

\section{DFT-Data}

Optimized geometry, SCF energy, three lower frequencies and thermochemistry for $\mathrm{I}_{\mathrm{H}} \quad 25$

Optimized geometry, SCF energy, three lower frequencies and thermochemistry for $\operatorname{IIb}_{\mathrm{H}} 26$

Optimized geometry, SCF energy, three lower frequencies and thermochemistry for $\mathrm{III}_{\mathrm{H}} \quad 27$

\section{Oniom-Data}

Optimized geometry, three lower frequencies and SCF energy for IIa 28

Optimized geometry, three lower frequencies and SCF energy for IIb 30

Complete Reference 16 
Experimental Data

NMR-Spectra of compound $\mathbf{2}$

${ }^{1}$ H-NMR (300MHz) THF-d8 of compound 2

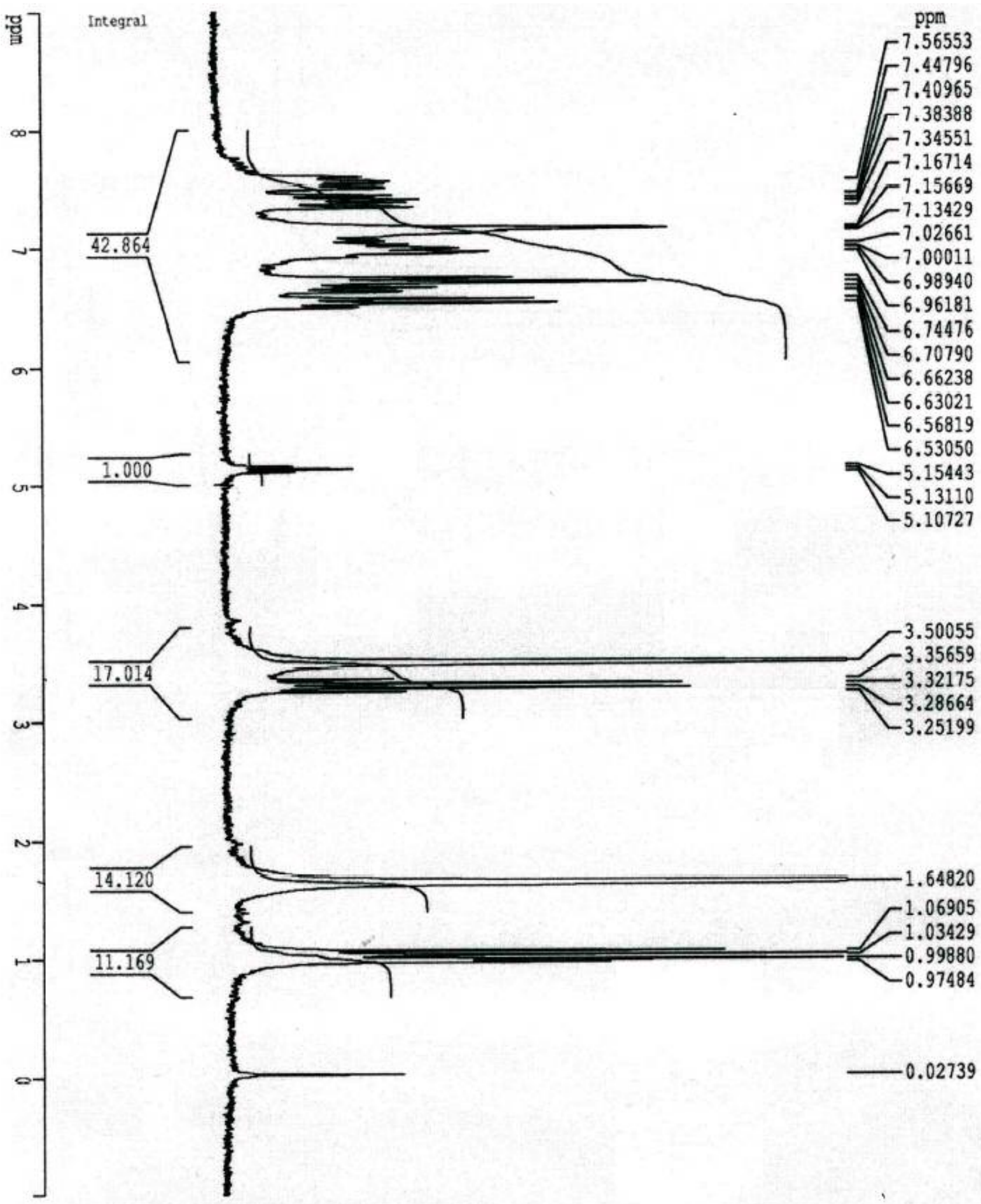


${ }^{13} \mathrm{C}-\mathrm{NMR}$ (300MHz) THF-d8 of compound 2

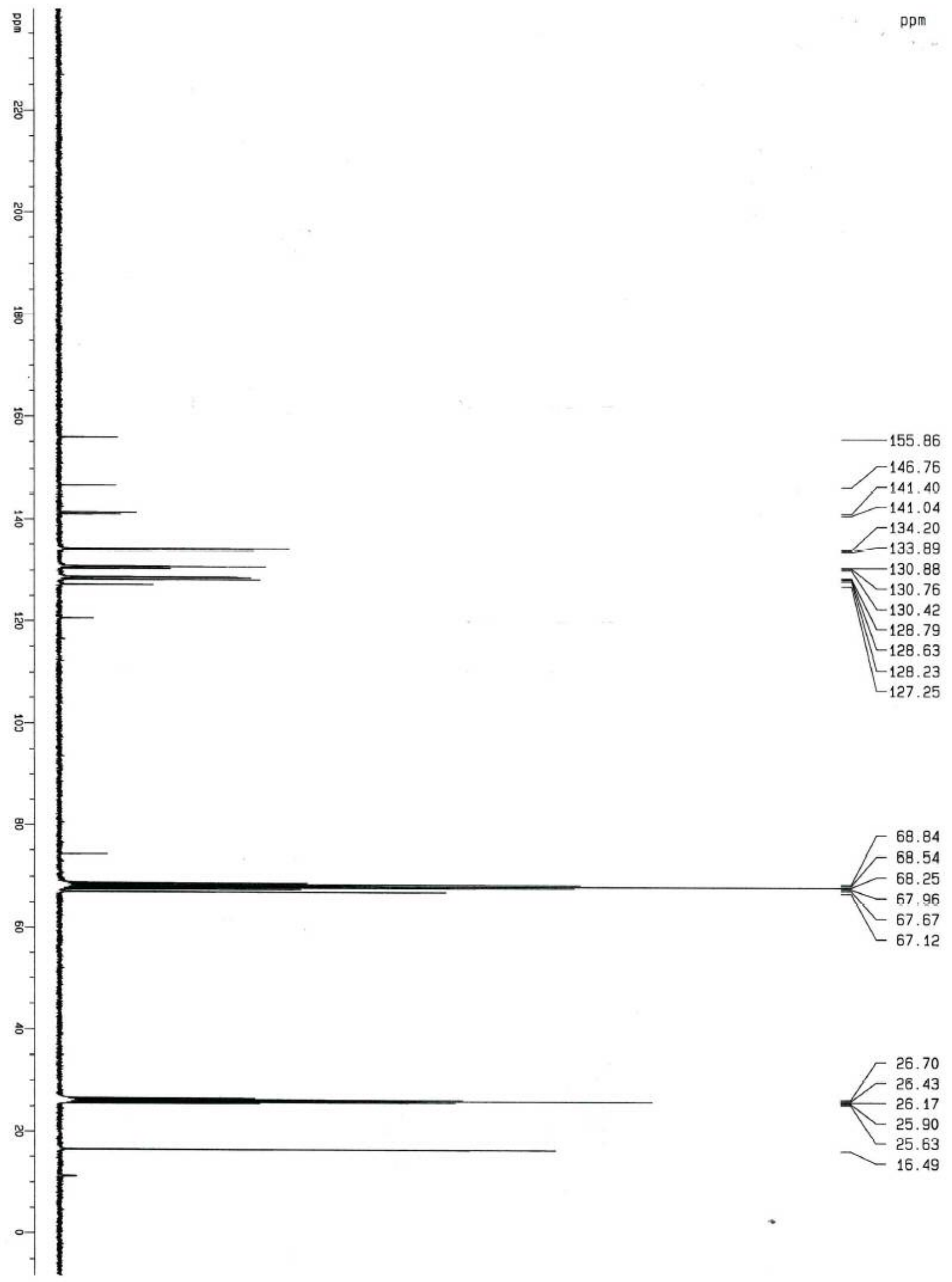


${ }^{31}$ P-NMR (300MHz) THF-d8 of compound 2

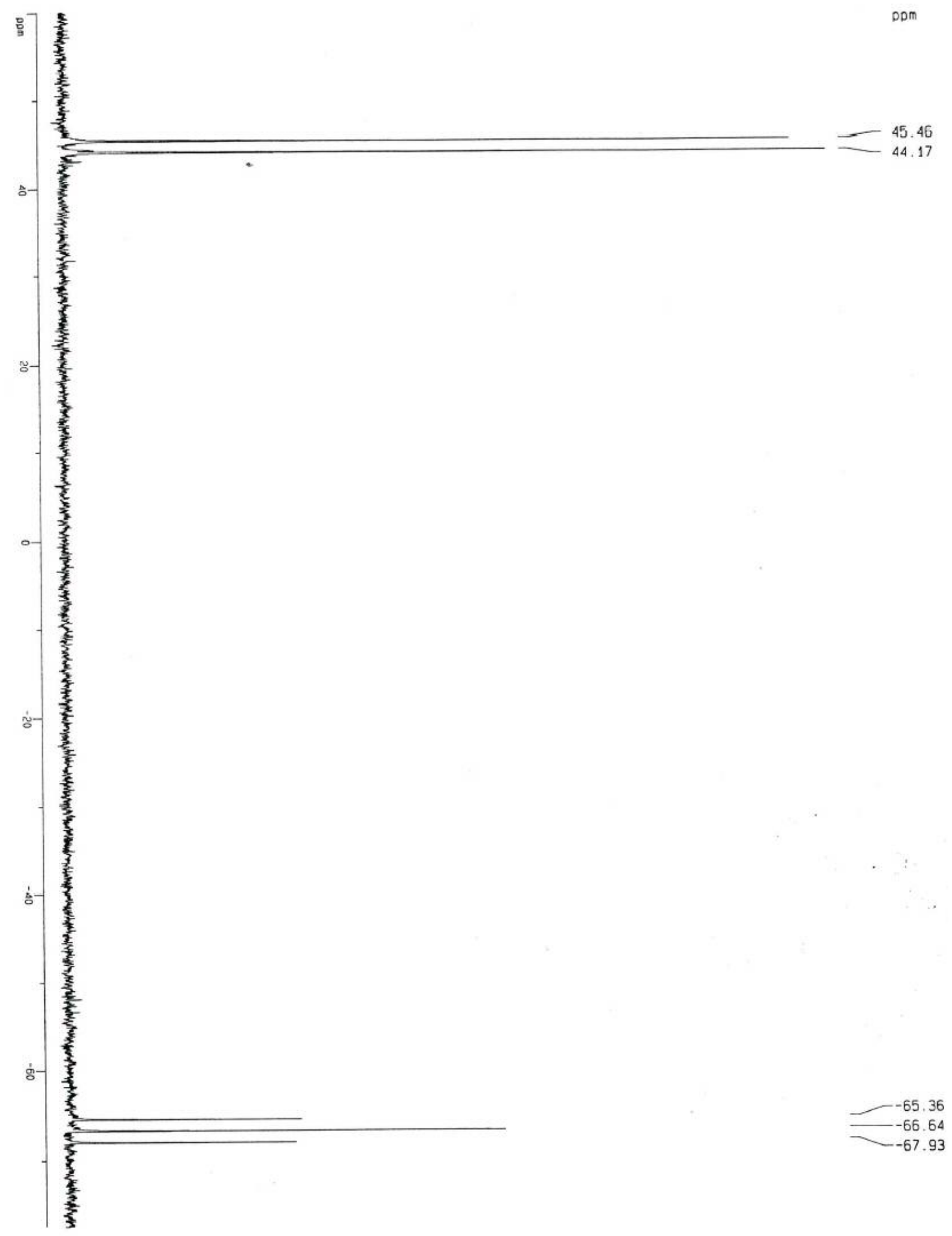

5 
NMR-Spectra of compound $\mathbf{3}$

${ }^{1}$ H-NMR (300MHz) THF-d8 of compound 3

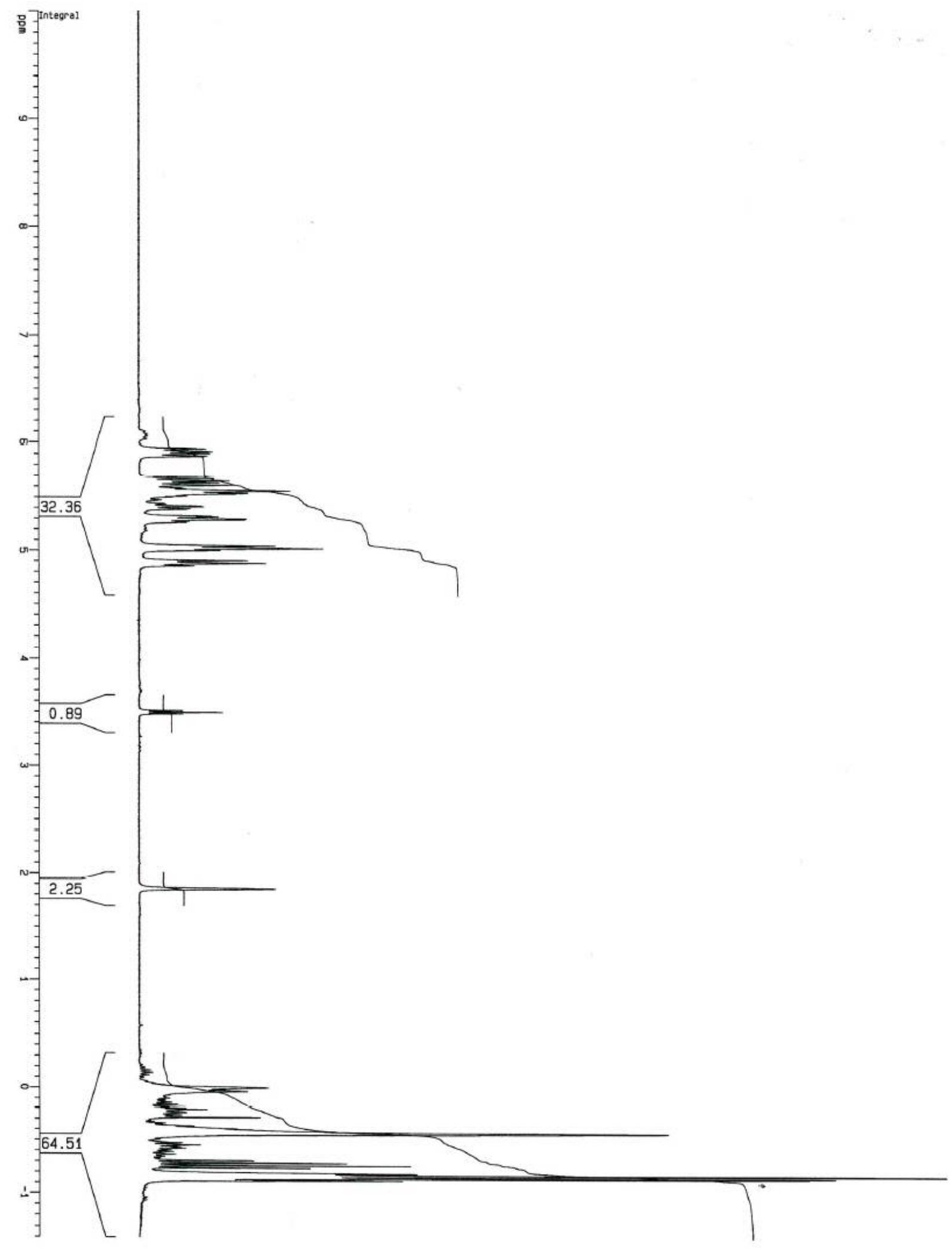

6 
${ }^{13} \mathrm{C}-\mathrm{NMR}$ (300MHz) THF-d8 of compound $\mathbf{3}$

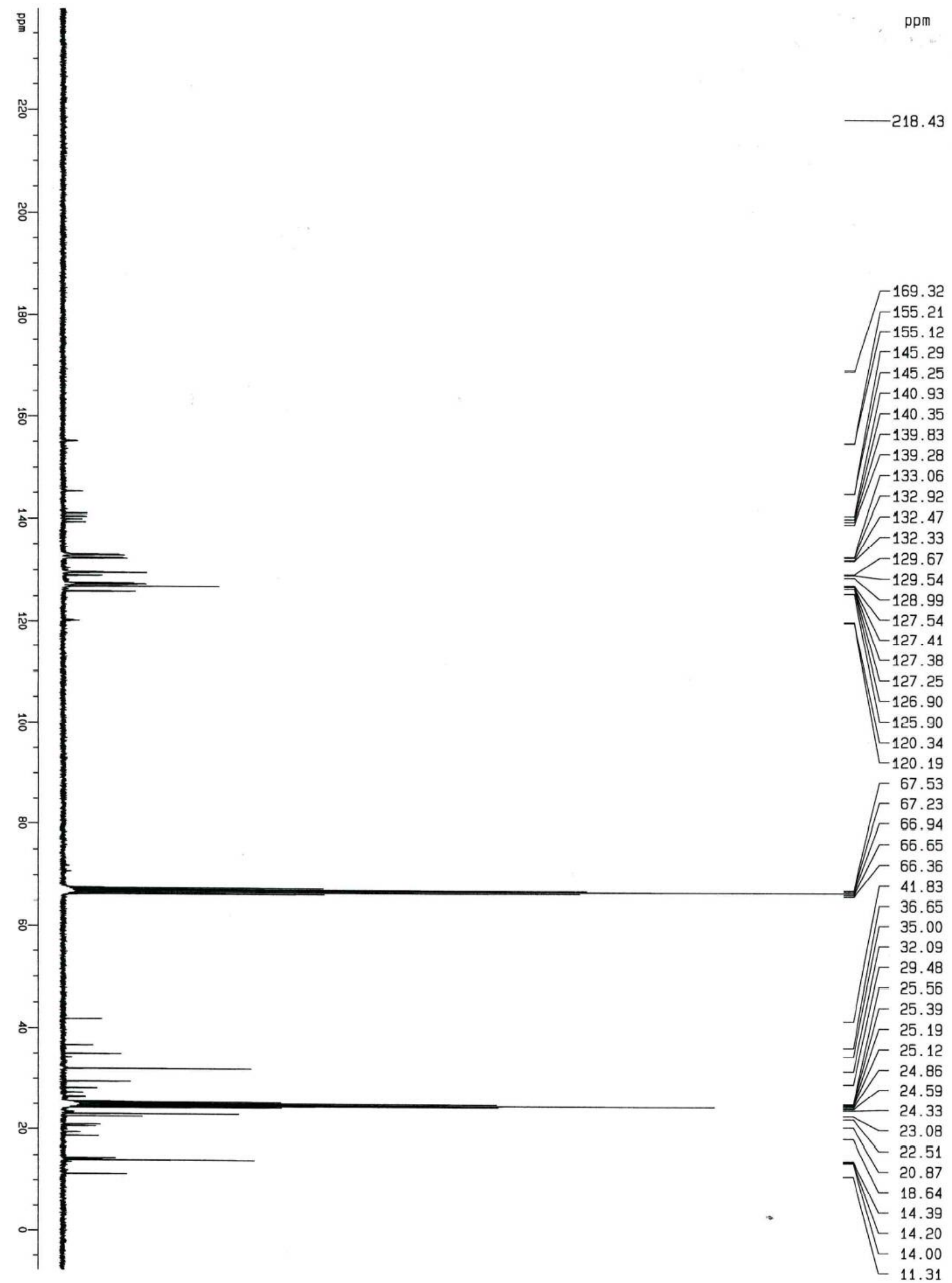


${ }^{31}$ P-NMR (300MHz) THF-d8 of compound 3

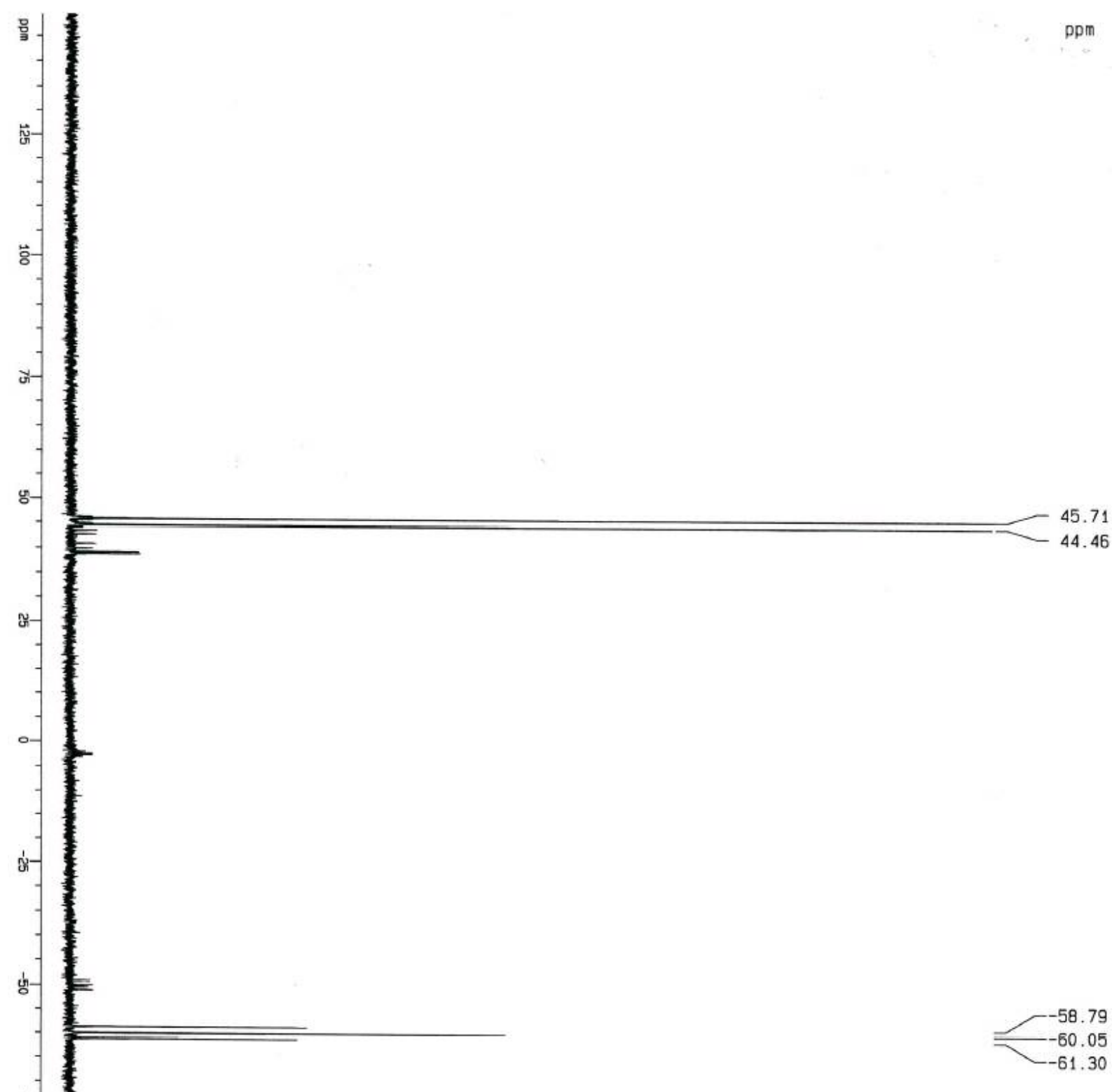

8 
NMR-Spectra of compounds $\mathbf{4}$ and $\mathbf{5}$

${ }^{1} \mathrm{H}-\mathrm{NMR}$ (300MHz) THF-d8 of compounds $\mathbf{4}$ and $\mathbf{5}$

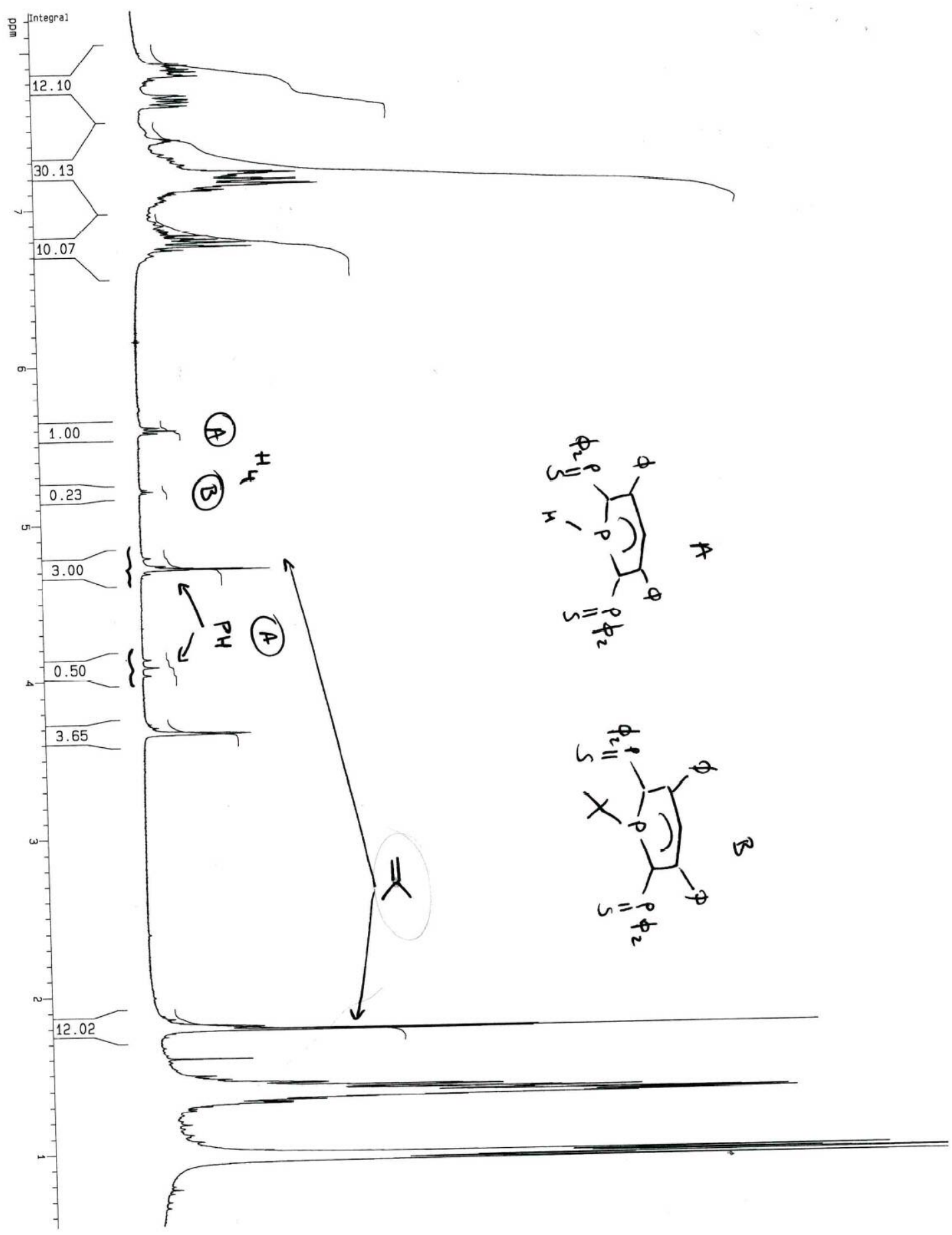


${ }^{13} \mathrm{C}-\mathrm{NMR}(300 \mathrm{MHz})$ THF-d8 of compounds $\mathbf{4}$ and $\mathbf{5}$

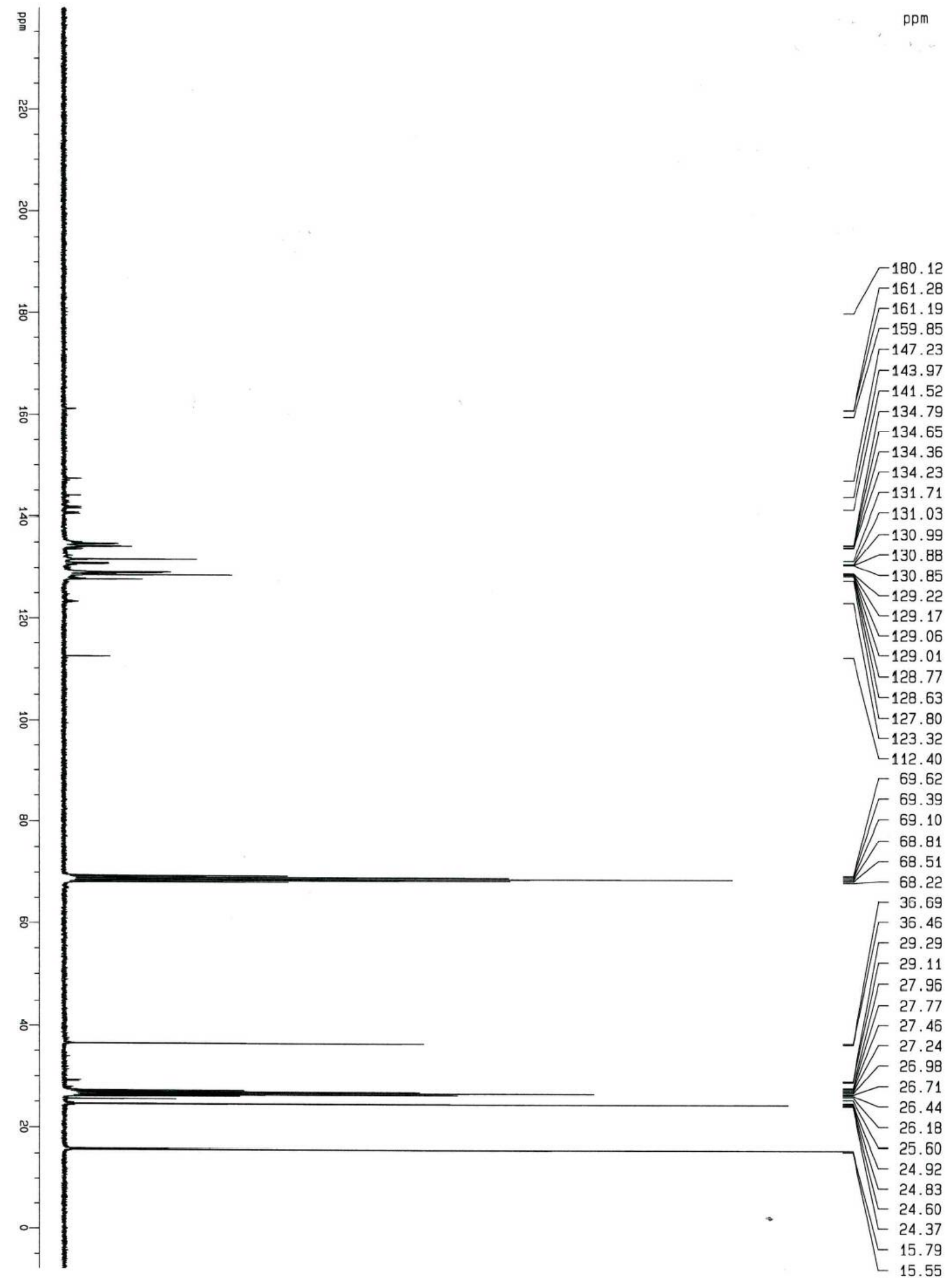


${ }^{31}$ P-NMR (300MHz) THF-d8 of compounds 4 and 5

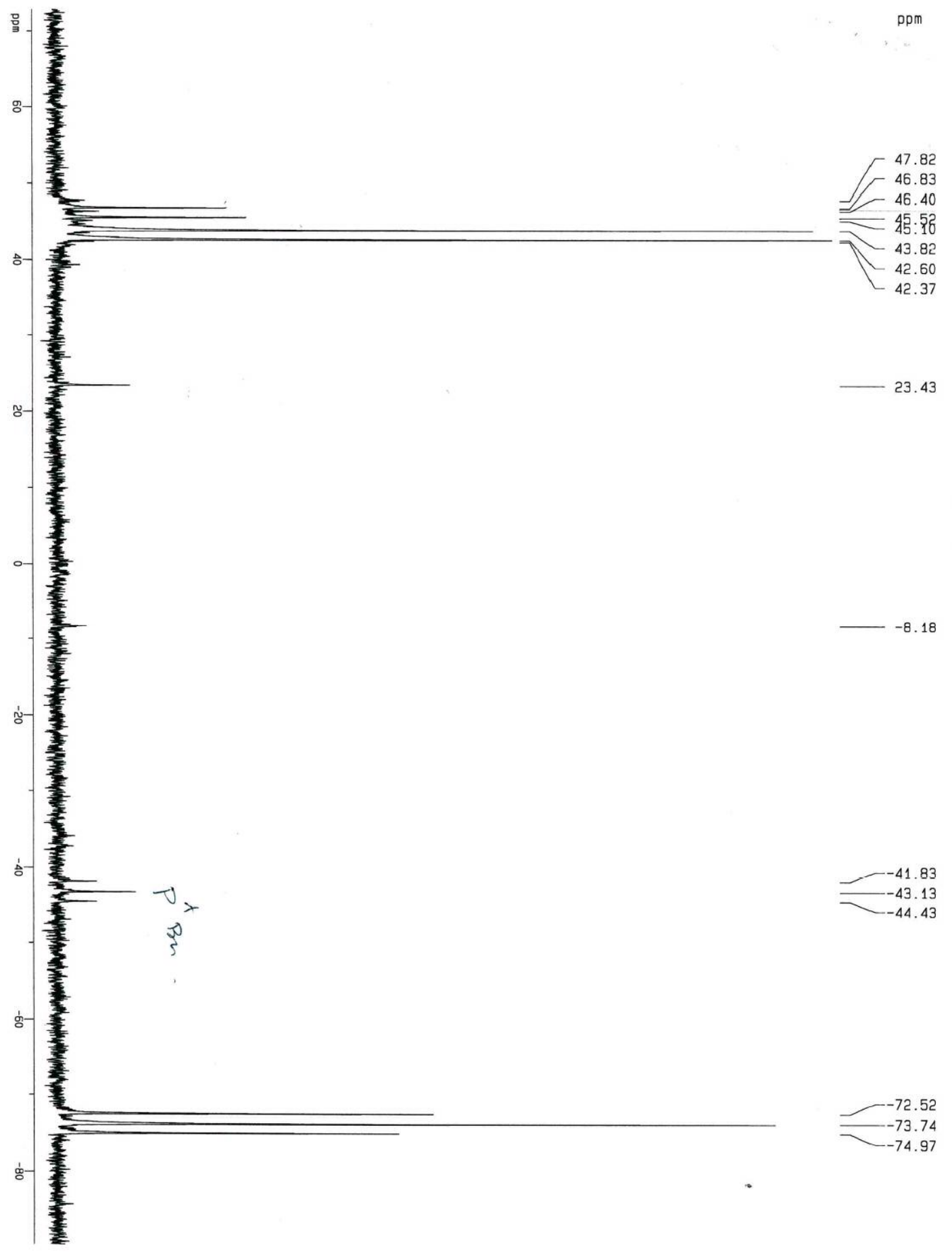


NMR-Spectrum of compound 7

${ }^{31}$ P-NMR (300MHz) THF-d8 of compound 7
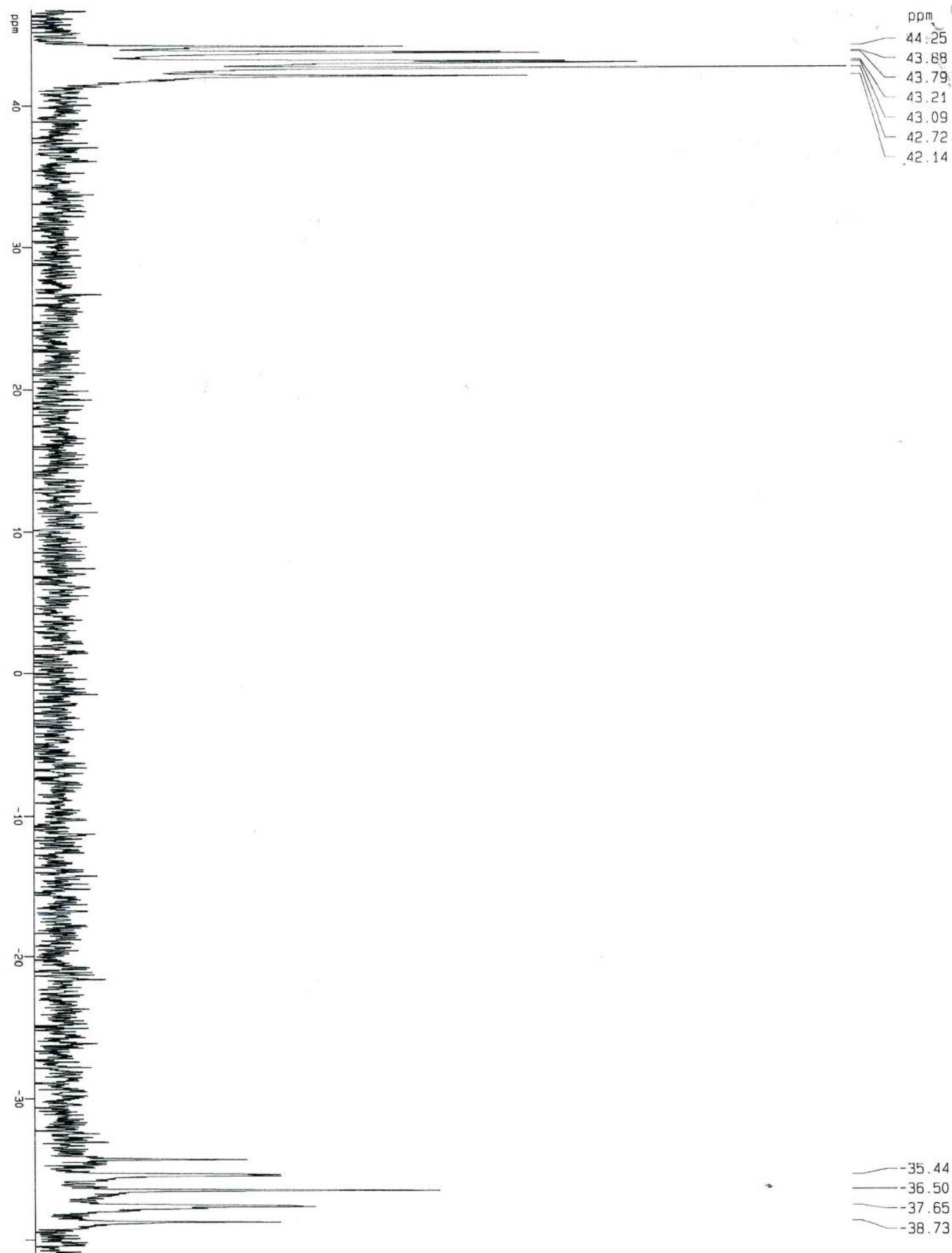
NMR-Spectra of compound 8a

${ }^{1}$ H-NMR (300MHz) THF-d8 of compound 8a

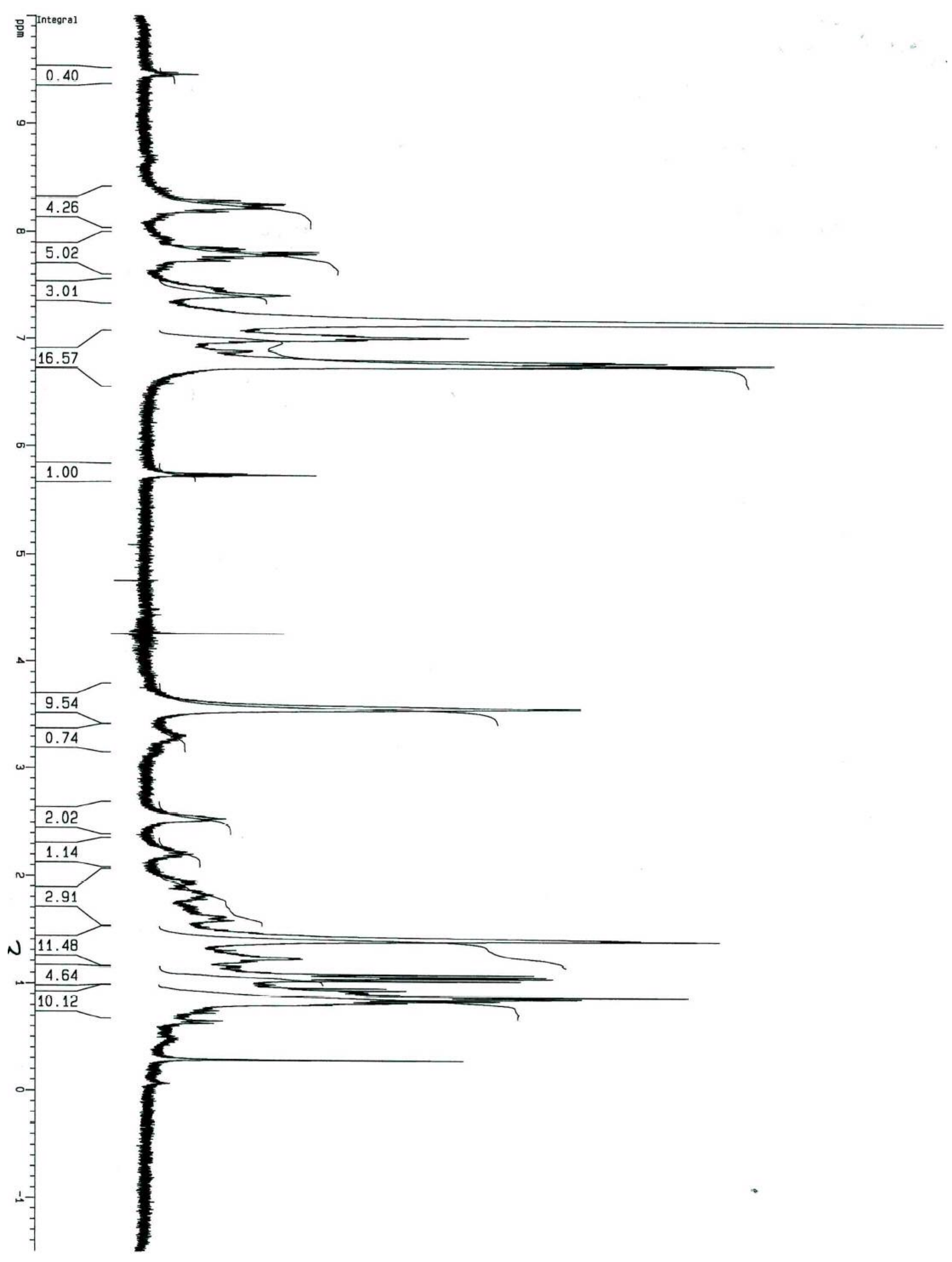

13 
${ }^{31}$ P-NMR (300MHz) THF-d8 of compound 8a

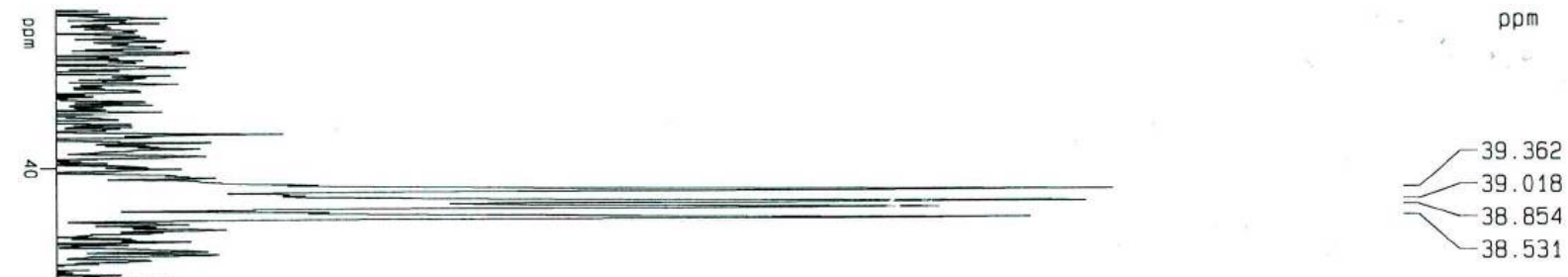

¿

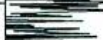

를

E

$=$

=

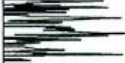

$\pm$

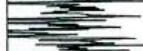

$\sqrt{2=}$

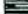

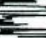



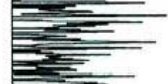

=

를

$\frac{2}{2=}$

-

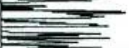

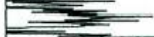
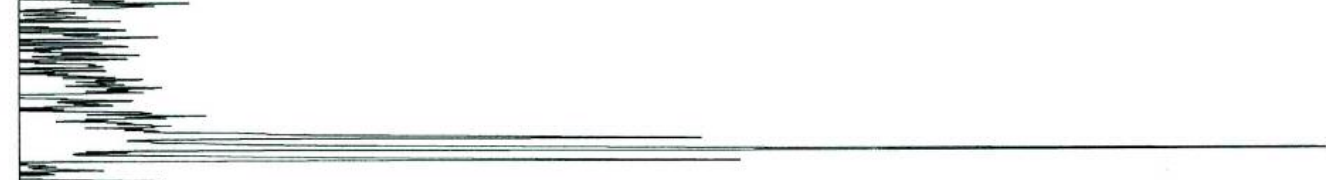

它-

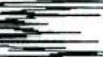


NMR-Spectrum of compounds $\mathbf{8 b}$ and $\mathbf{8 c}$

${ }^{31} \mathrm{P}-\mathrm{NMR}$ (300MHz) THF-d8 of compounds $\mathbf{8 b}$ and $\mathbf{8 c}$

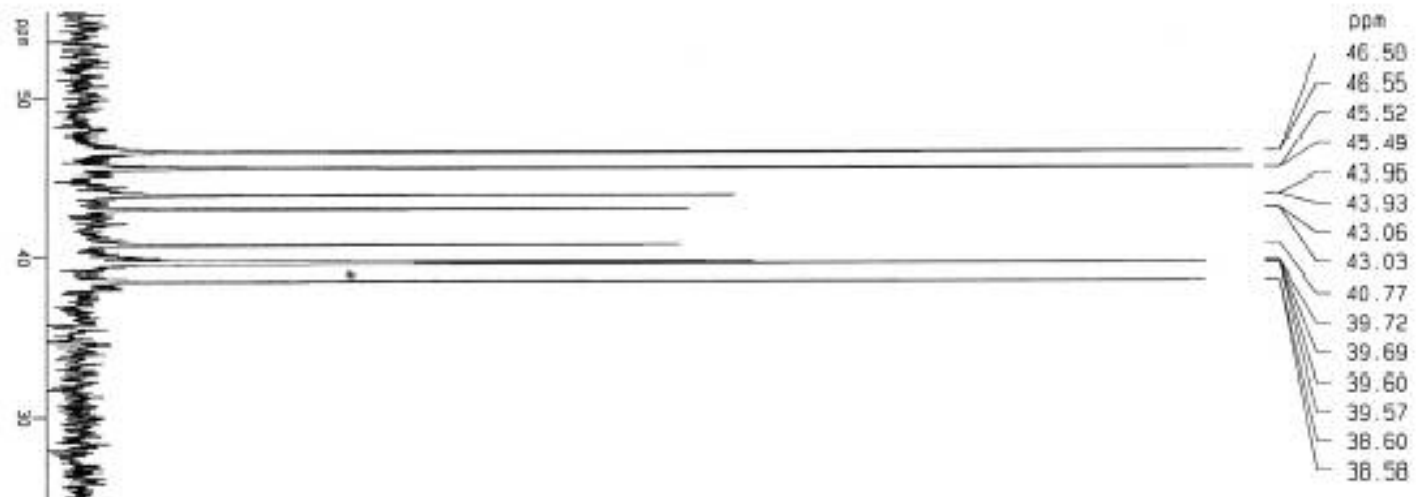

ㄴ-

:

-

b-

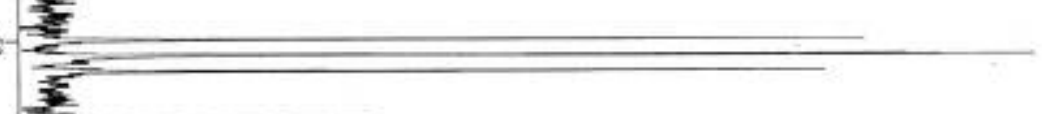


NMR-Spectrum of compound 9

${ }^{31}$ P-NMR (300MHz) THF-d8 of compound 9

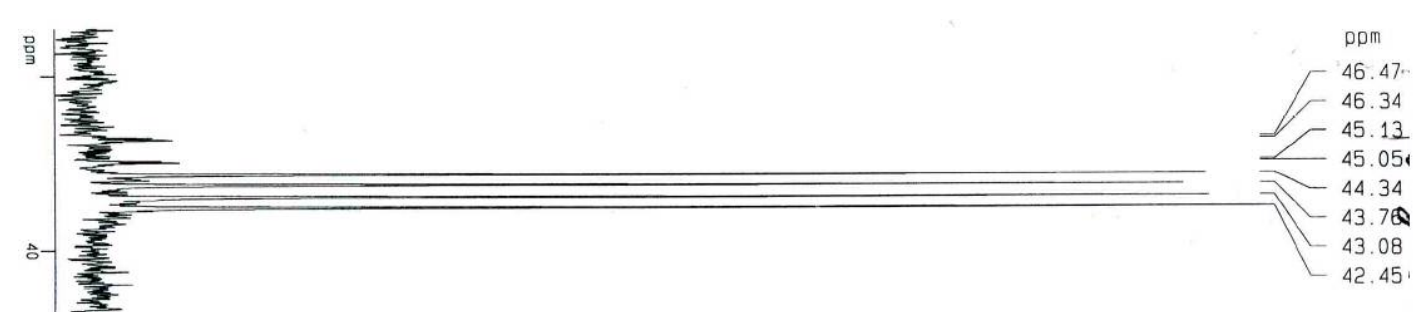

今́ 
NMR-Spectra of compound $\mathbf{1 0}$

${ }^{1} \mathrm{H}-\mathrm{NMR}(300 \mathrm{MHz})$ THF-d8 of compound 10

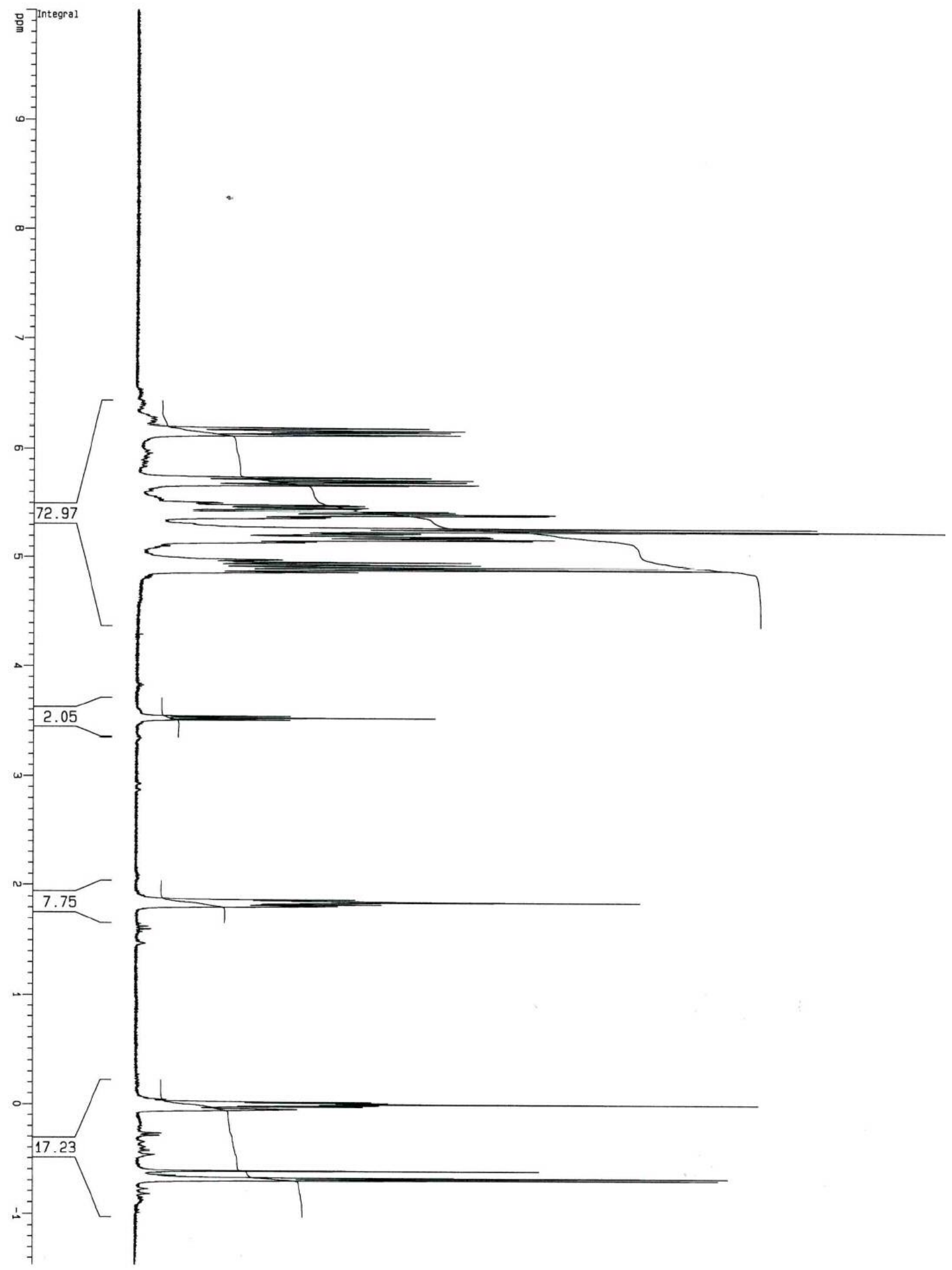


${ }^{13} \mathrm{C}-\mathrm{NMR}(300 \mathrm{MHz})$ THF-d8 of compound $\mathbf{1 0}$

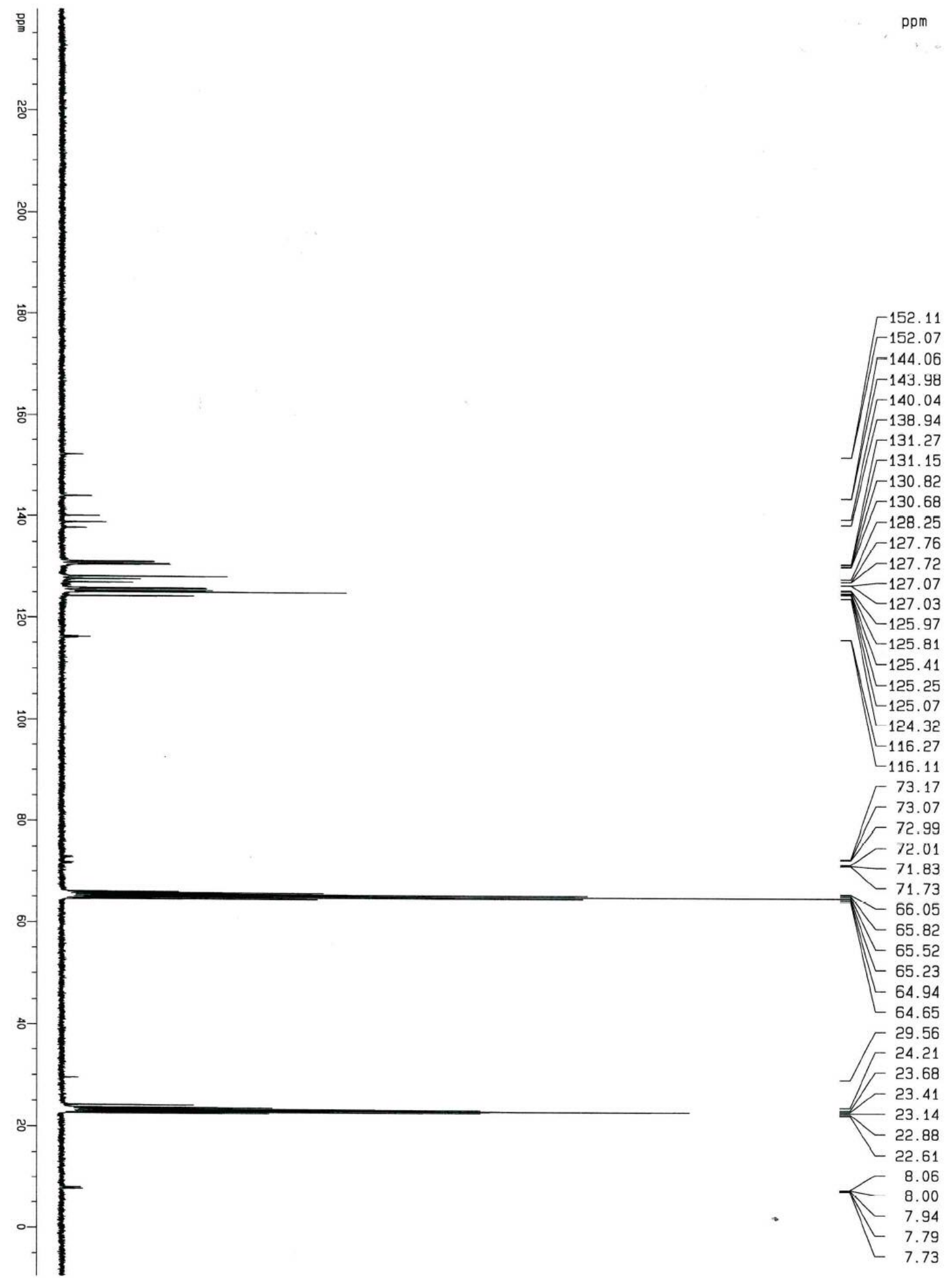


${ }^{31}$ P-NMR (300MHz) THF-d8 of compound 10

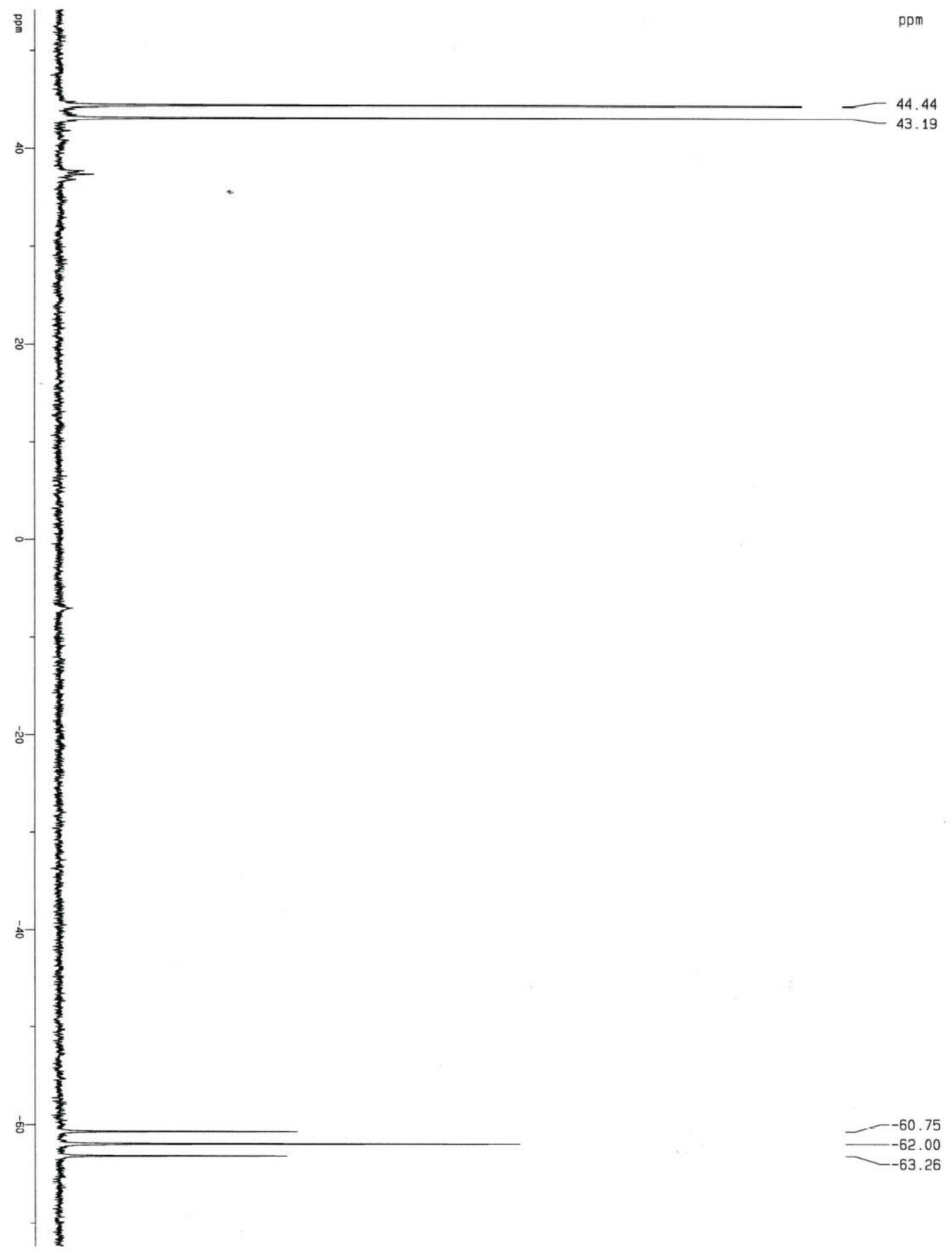


NMR-Spectra of compound $\mathbf{1 1}$

${ }^{1}$ H-NMR (300MHz) THF-d8 of compound 11

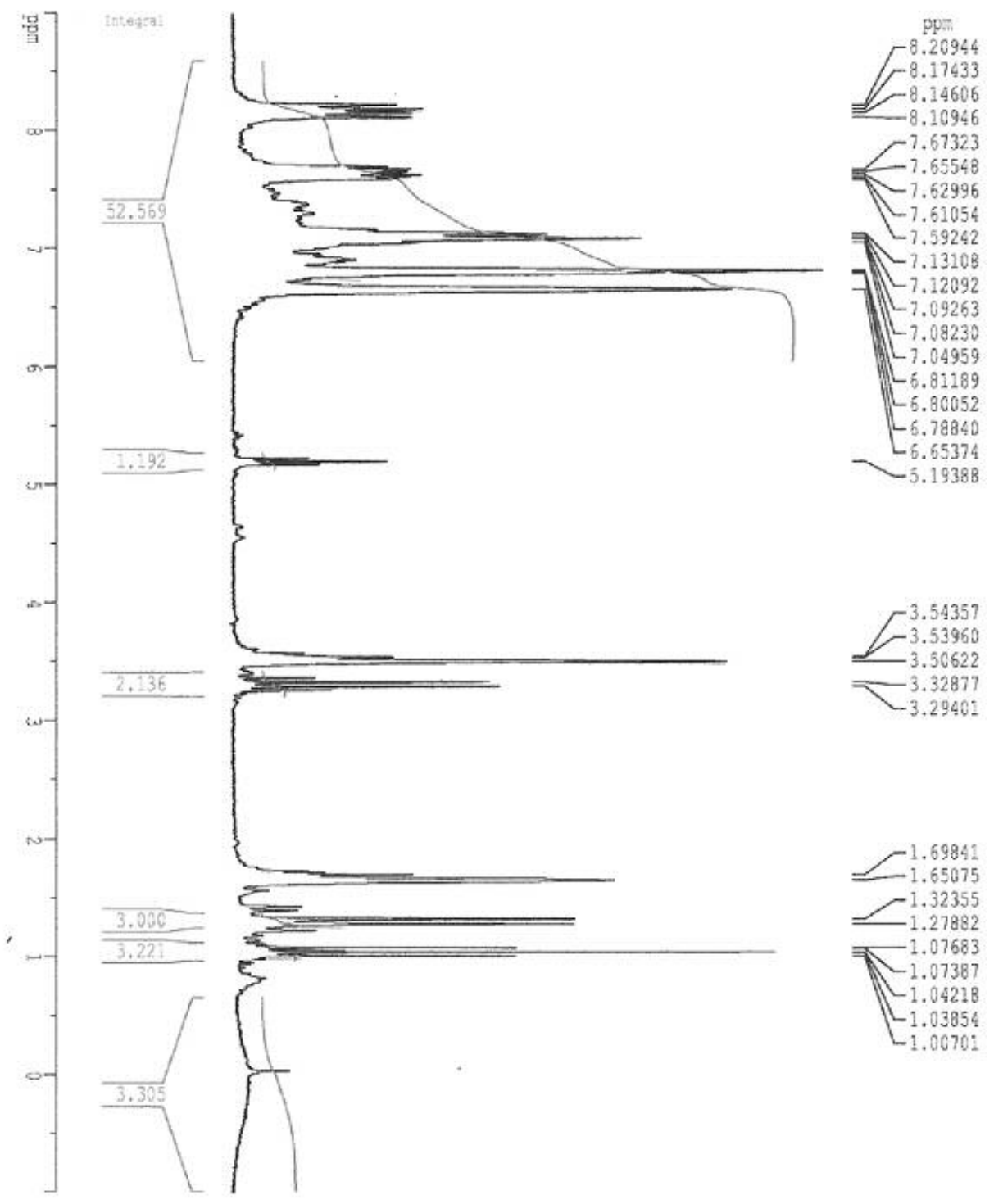


${ }^{31}$ P-NMR (300MHz) THF-d8 of compound 11

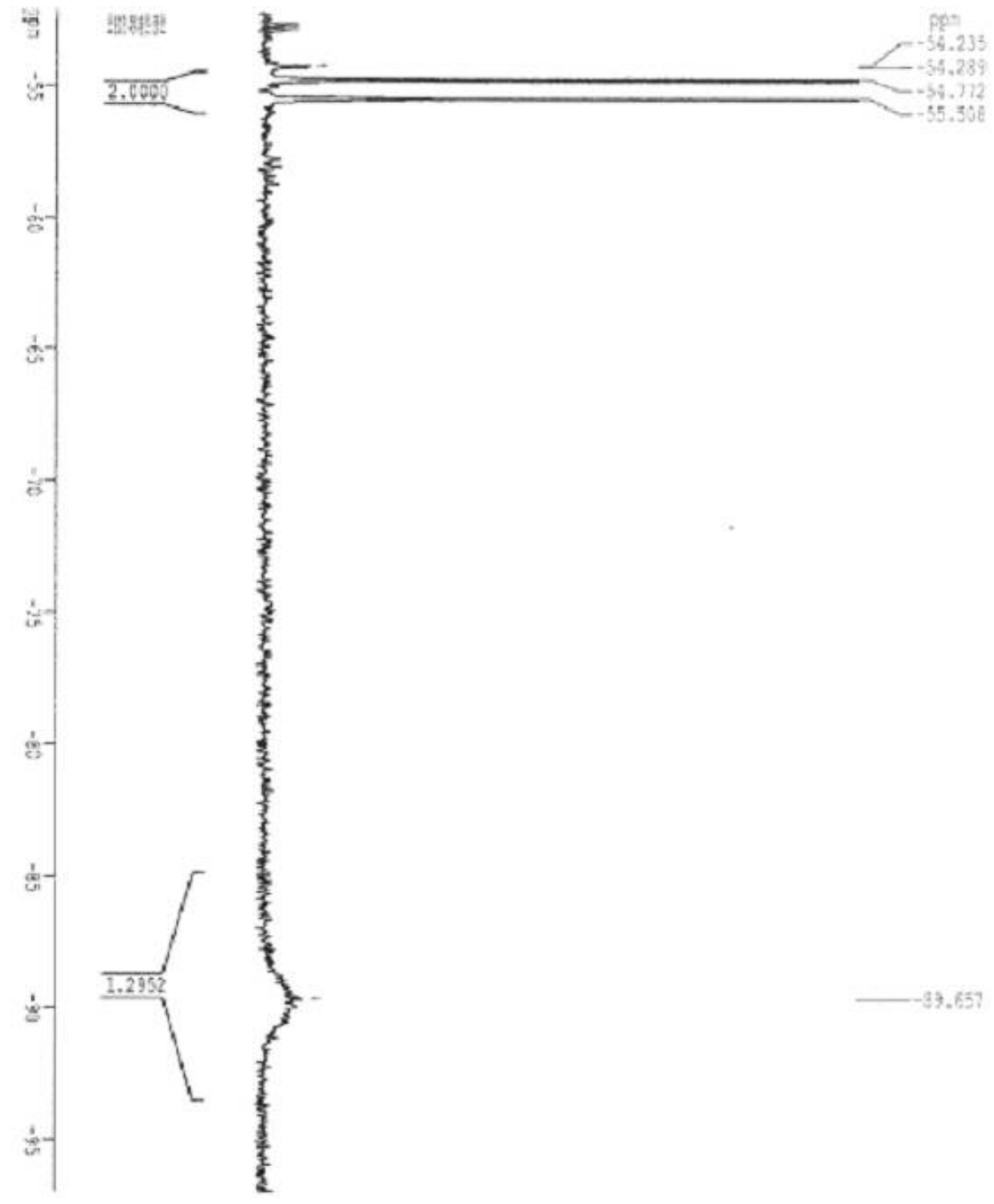


NMR-Spectra of compound $\mathbf{1 2}$

${ }^{1}$ H-NMR (300MHz) THF-d8 of compound 12

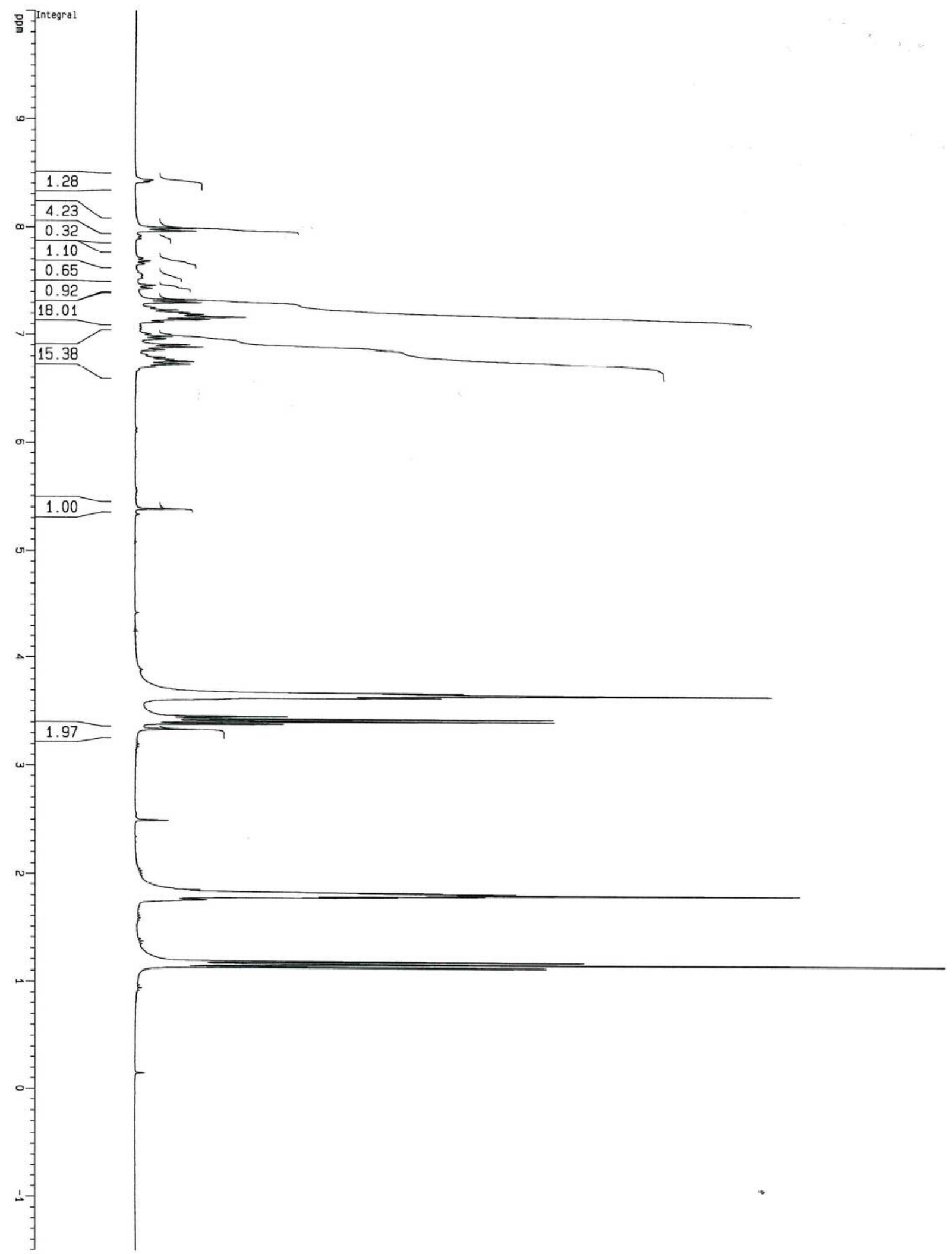


${ }^{13} \mathrm{C}$-NMR (300MHz) THF-d8 of compound 12

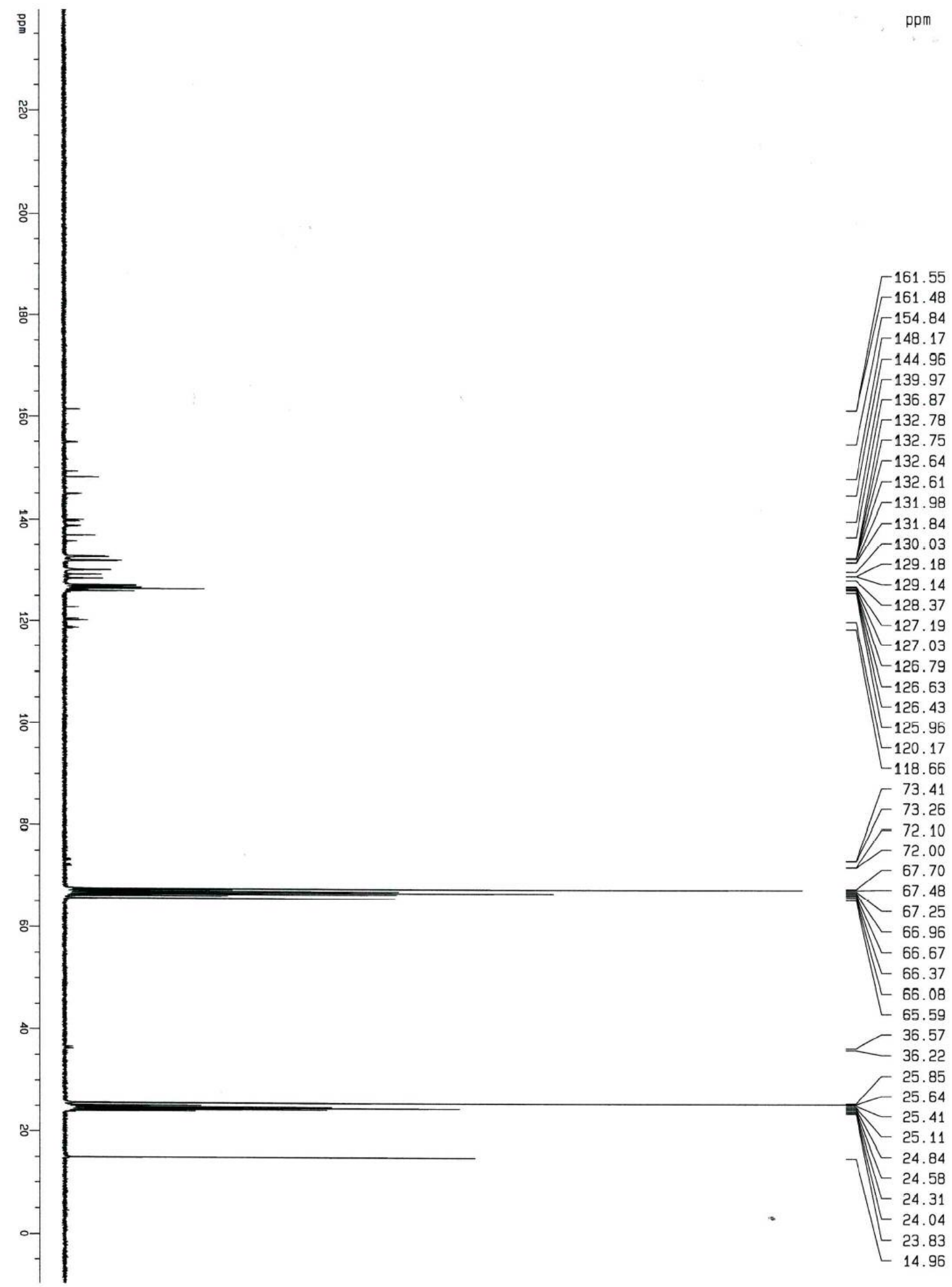


${ }^{31}$ P-NMR (300MHz) THF-d8 of compound 12
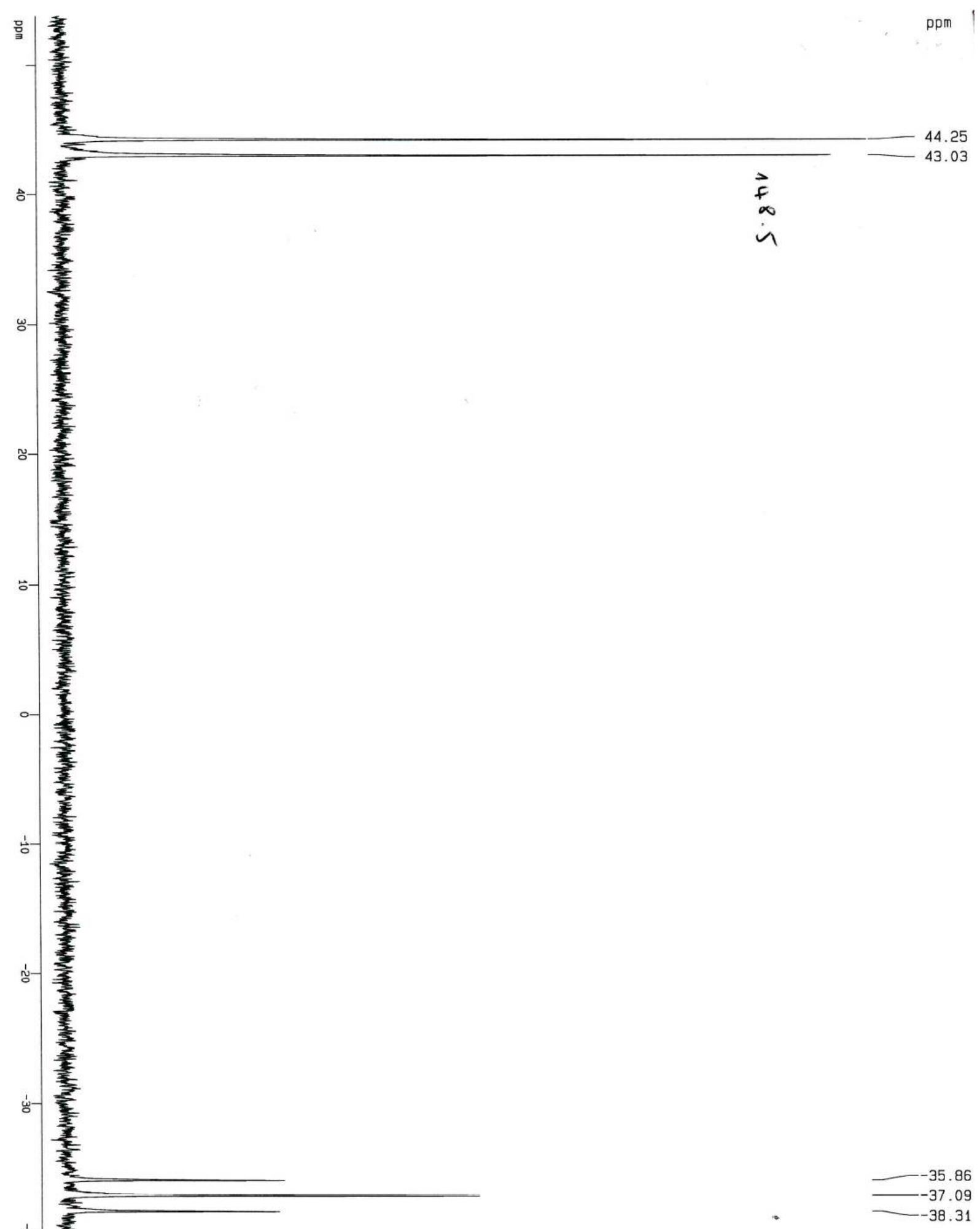

24 


\section{DFT-Data}

Optimized geometry, SCF energy, three lower frequencies and thermochemistry for $\mathrm{I}_{\mathrm{H}}$

\begin{tabular}{|c|c|c|c|c|c|}
\hline $\begin{array}{l}\text { Center } \\
\text { Number }\end{array}$ & $\begin{array}{l}\text { Atomic } \\
\text { Number }\end{array}$ & $\begin{array}{r}\text { Atomic } \\
\text { Type }\end{array}$ & $\begin{array}{l}\text { Coord } \\
\mathrm{X}\end{array}$ & $\begin{aligned} \text { rates (Angs } & \text { (A } \\
& \end{aligned}$ & $\begin{array}{ll}\text { roms ) } & \\
& \mathrm{Z}\end{array}$ \\
\hline 1 & 6 & 0 & -0.000270 & 0.000246 & -0.000699 \\
\hline 2 & 6 & 0 & -0.000264 & 0.000096 & 1.395152 \\
\hline 3 & 6 & 0 & 2.688067 & 0.000189 & 1.162747 \\
\hline 4 & 6 & 0 & 2.448483 & 0.000236 & -0.212447 \\
\hline 5 & 6 & 0 & 1.166744 & 0.000252 & -0.769697 \\
\hline 6 & 1 & 0 & -0.962939 & 0.001339 & -0.511258 \\
\hline 7 & 1 & 0 & 3.309136 & 0.001172 & -0.880844 \\
\hline 8 & 1 & 0 & 1.073133 & 0.000823 & -1.852322 \\
\hline 9 & 15 & 0 & 1.439978 & 0.000879 & 2.391274 \\
\hline 10 & 15 & 0 & 4.445529 & -0.000774 & 1.734515 \\
\hline 11 & 1 & 0 & 4.463048 & 1.075277 & 2.653569 \\
\hline 12 & 1 & 0 & 4.469770 & -1.094851 & 2.631979 \\
\hline 13 & 16 & 0 & 5.843201 & 0.016036 & 0.356209 \\
\hline 14 & 15 & 0 & -1.633842 & -0.001511 & 2.259393 \\
\hline 15 & 1 & 0 & -1.505614 & -1.098114 & 3.144846 \\
\hline 16 & 1 & 0 & -1.492548 & 1.071642 & 3.171094 \\
\hline 17 & 16 & 0 & -3.246802 & 0.020248 & 1.140642 \\
\hline
\end{tabular}

$\mathrm{HF}=-2015.1811387$

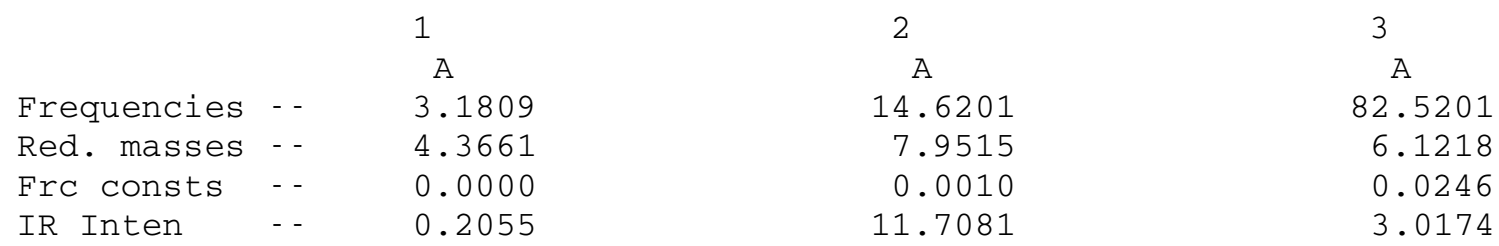

Sum of electronic and zero-point Energies=

$-2015.073728$

Sum of electronic and thermal Enthalpies=

$-2015.061268$

Sum of electronic and thermal Free Energies=

$-2015.116904$ 
Optimized geometry, SCF energy, three lower frequencies and thermochemistry for $\mathrm{IIb}_{\mathrm{H}}$

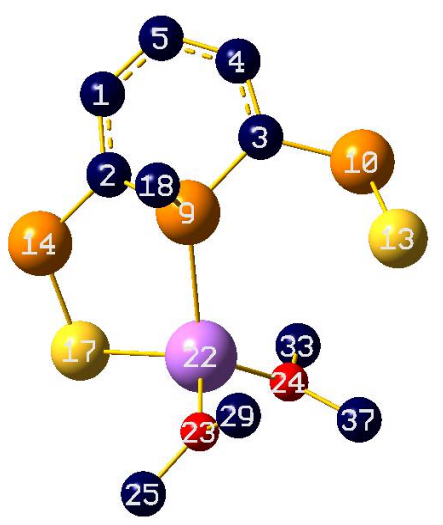

\begin{tabular}{|c|c|c|c|c|c|}
\hline \multirow{2}{*}{$\begin{array}{l}\text { Center } \\
\text { Number }\end{array}$} & \multirow{2}{*}{$\begin{array}{l}\text { Atomic } \\
\text { Number }\end{array}$} & \multirow{2}{*}{$\begin{array}{r}\text { Atomic } \\
\text { Type }\end{array}$} & \multicolumn{3}{|c|}{ Coordinates (Angstroms) } \\
\hline & & & $\mathrm{X}$ & Y & Z \\
\hline$-\cdots$ & & - - - - & $-\ldots-\ldots$ & $-\ldots \ldots$ & $\ldots \ldots$ \\
\hline 1 & 6 & 0 & 0.002818 & 0.020715 & 0.003396 \\
\hline 2 & 6 & 0 & 0.002036 & 0.005623 & 1.401922 \\
\hline 3 & 6 & 0 & 2.740098 & 0.001402 & 1.121083 \\
\hline 4 & 6 & 0 & 2.444547 & 0.027330 & -0.235704 \\
\hline 5 & 6 & 0 & 1.151763 & 0.144102 & -0.788891 \\
\hline 6 & 1 & 0 & -0.940032 & -0.137240 & -0.526046 \\
\hline 7 & 1 & 0 & 3.261276 & -0.128284 & -0.945752 \\
\hline 8 & 1 & 0 & 1.045992 & 0.154543 & -1.869467 \\
\hline 9 & 15 & 0 & 1.483773 & 0.463362 & 2.355431 \\
\hline 10 & 15 & 0 & 4.356885 & -0.506010 & 1.674261 \\
\hline 11 & 1 & 0 & 4.358532 & -1.920687 & 1.818667 \\
\hline 12 & 1 & 0 & 5.135041 & -0.402924 & 0.493471 \\
\hline 13 & 16 & 0 & 5.135143 & 0.358571 & 3.287871 \\
\hline 14 & 15 & 0 & -1.438464 & -0.467269 & 2.305411 \\
\hline 15 & 1 & 0 & -2.242368 & 0.659850 & 2.618417 \\
\hline 16 & 1 & 0 & -2.290829 & -1.104964 & 1.372602 \\
\hline 17 & 16 & 0 & -1.175170 & -1.543198 & 3.993446 \\
\hline 18 & 6 & 0 & 1.510060 & 2.331724 & 2.258520 \\
\hline 19 & 1 & 0 & 2.466288 & 2.689667 & 2.657411 \\
\hline 20 & 1 & 0 & 1.400570 & 2.677059 & 1.225202 \\
\hline 21 & 1 & 0 & 0.694255 & 2.746026 & 2.864705 \\
\hline 22 & 3 & 0 & 1.154814 & -0.834882 & 4.552052 \\
\hline 23 & 8 & 0 & 1.358109 & 0.314397 & 6.125843 \\
\hline 24 & 8 & 0 & 2.218608 & -2.451303 & 4.825724 \\
\hline 25 & 6 & 0 & 0.325652 & 0.475860 & 7.097959 \\
\hline 26 & 1 & 0 & 0.743144 & 0.413260 & 8.112263 \\
\hline 27 & 1 & 0 & -0.175302 & 1.444978 & 6.968744 \\
\hline 28 & 1 & 0 & -0.396969 & -0.326992 & 6.941031 \\
\hline 29 & 6 & 0 & 2.353048 & 1.341710 & 6.185837 \\
\hline 30 & 1 & 0 & 3.090450 & 1.129650 & 5.408759 \\
\hline 31 & 1 & 0 & 1.896948 & 2.324148 & 6.003912 \\
\hline 32 & 1 & 0 & 2.837811 & 1.341448 & 7.171711 \\
\hline 33 & 6 & 0 & 2.104819 & -3.574424 & 3.950620 \\
\hline 34 & 1 & 0 & 1.170784 & -3.455130 & 3.399100 \\
\hline 35 & 1 & 0 & 2.953650 & -3.607554 & 3.254015 \\
\hline 36 & 1 & 0 & 2.073911 & -4.505509 & 4.532797 \\
\hline 37 & 6 & 0 & 3.397209 & -2.487668 & 5.632988 \\
\hline 38 & 1 & 0 & 4.296202 & -2.393213 & 5.011490 \\
\hline 39 & 1 & 0 & 3.342258 & -1.639202 & 6.316709 \\
\hline
\end{tabular}


$\mathrm{HF}=-2372.7845752$

\begin{tabular}{|c|c|c|c|}
\hline & 1 & 2 & 3 \\
\hline & A & A & $\mathrm{A}$ \\
\hline Frequencies & 20.9219 & 30.8891 & 38.2339 \\
\hline Red. masses & 5.2118 & 2.7946 & 4.2307 \\
\hline Frc consts & 0.0013 & 0.0016 & 0.0036 \\
\hline IR Inten & 1.8851 & 0.2990 & 0.6662 \\
\hline
\end{tabular}

Sum of electronic and zero-point Energies=

Sum of electronic and thermal Enthalpies=

$-2372.474783$

sum of electronic and thermal Free Energies=

$-2372.447056$

$-2372.535148$

Optimized geometry, SCF energy, three lower frequencies and thermochemistry for $\mathrm{III}_{\mathrm{H}}$

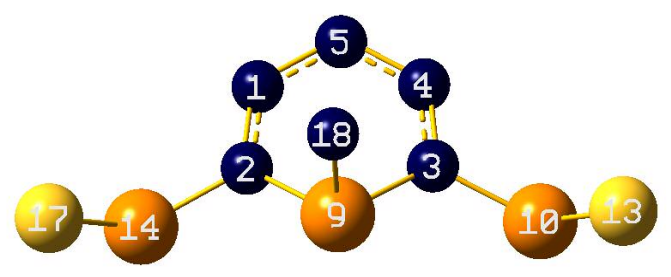

\begin{tabular}{ccrrrr}
$\begin{array}{c}\text { Center } \\
\text { Number }\end{array}$ & $\begin{array}{c}\text { Atomic } \\
\text { Number }\end{array}$ & $\begin{array}{c}\text { Atomic } \\
\text { Type }\end{array}$ & \multicolumn{2}{c}{$\begin{array}{c}\text { Coordinates } \\
\text { X Angstroms) }\end{array}$} \\
\hline 1 & 6 & 0 & -0.000713 & 0.002250 & 0.002089 \\
2 & 6 & 0 & -0.000619 & 0.004303 & 1.393837 \\
3 & 6 & 0 & 2.725402 & 0.002853 & 1.108658 \\
4 & 6 & 0 & 2.437200 & 0.000782 & -0.252971 \\
5 & 6 & 0 & 1.146996 & 0.101945 & -0.806666 \\
6 & 1 & 0 & -0.947971 & -0.167914 & -0.518063 \\
7 & 1 & 0 & 3.256138 & -0.170331 & -0.957894 \\
8 & 1 & 0 & 1.034000 & 0.065437 & -1.887188 \\
9 & 15 & 0 & 1.478630 & 0.505635 & 2.359779 \\
10 & 15 & 0 & 4.363182 & -0.411079 & 1.654679 \\
11 & 1 & 0 & 4.156399 & -1.362520 & 2.683497 \\
12 & 1 & 0 & 4.852638 & -1.242505 & 0.611918 \\
13 & 16 & 0 & 5.712219 & 0.958989 & 2.200544 \\
14 & 15 & 0 & -1.488431 & -0.411518 & 2.268683 \\
15 & 1 & 0 & -2.179665 & -1.251330 & 1.354725 \\
16 & 1 & 0 & -1.067125 & -1.355571 & 3.236921 \\
17 & 16 & 0 & -2.702710 & 0.956532 & 3.074458 \\
18 & 6 & 0 & 1.458698 & 2.372287 & 2.138131 \\
19 & 1 & 0 & 2.399621 & 2.780880 & 2.526436 \\
20 & 1 & 0 & 1.348860 & 2.657725 & 1.085982 \\
21 & 1 & 0.621175 & 2.785473 & 2.713280
\end{tabular}

$\mathrm{HF}=-2055.1630024$

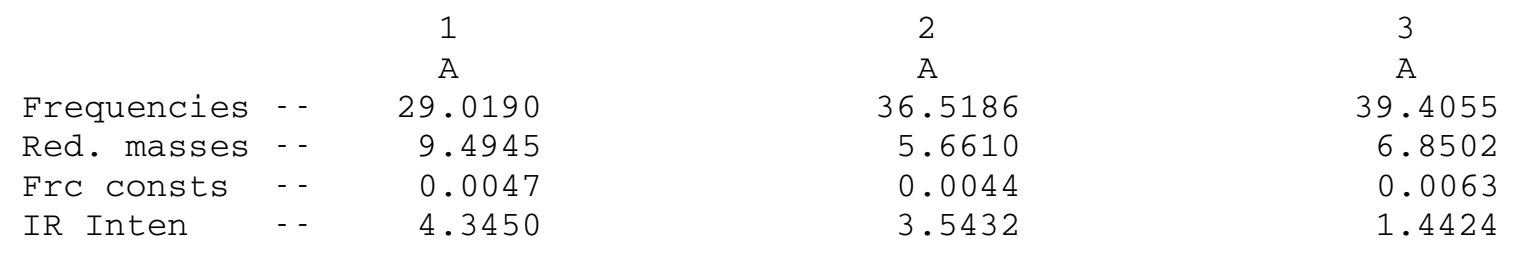


Sum of electronic and zero-point Energies=

$-2055.019904$

Sum of electronic and thermal Enthalpies=

$-2055.005227$

Sum of electronic and thermal Free Energies=

$-2055.062991$

\section{Oniom-Data}

Optimized geometry, three lower frequencies and SCF energy for IIa

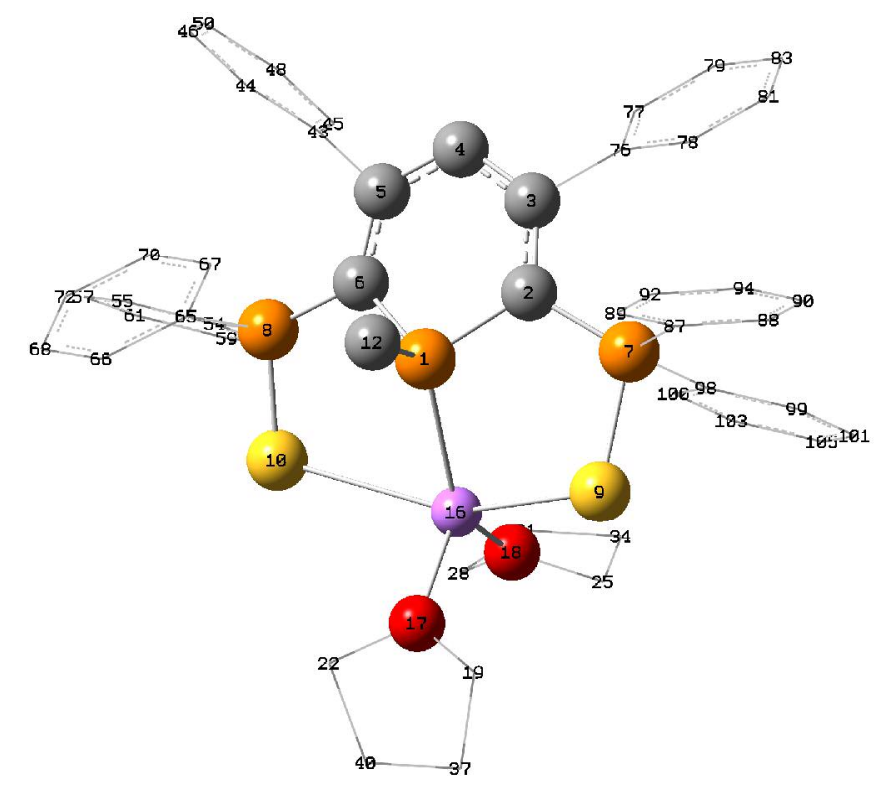

\begin{tabular}{|c|c|c|c|c|c|}
\hline \multirow{2}{*}{$\begin{array}{l}\text { Center } \\
\text { Number }\end{array}$} & \multirow{2}{*}{$\begin{array}{l}\text { Atomic } \\
\text { Number }\end{array}$} & \multirow{2}{*}{$\begin{array}{l}\text { Atomic } \\
\text { Type }\end{array}$} & \multicolumn{3}{|c|}{ Coordinates (Angstroms) } \\
\hline & & & $\mathrm{X}$ & $\mathrm{Y}$ & Z \\
\hline & & & & 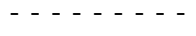 & $\cdots$ \\
\hline 1 & 15 & 10151003 & -0.115783 & 0.254568 & -1.406218 \\
\hline 2 & 6 & 10061000 & 1.441998 & -0.584363 & -0.999816 \\
\hline 3 & 6 & 10061000 & 1.477989 & -1.978775 & -1.123471 \\
\hline 4 & 6 & 10061000 & 0.317330 & -2.761594 & -1.311624 \\
\hline 5 & 6 & 10061000 & -0.979230 & -2.336547 & -0.965723 \\
\hline 6 & 6 & 10061000 & -1.268472 & -0.974384 & -0.753514 \\
\hline 7 & 15 & 10151004 & 2.602522 & 0.499466 & -0.171368 \\
\hline 8 & 15 & 10151004 & -2.393401 & -0.234966 & 0.417863 \\
\hline 9 & 16 & 10161004 & 2.336718 & 2.436631 & -0.622241 \\
\hline 10 & 16 & 10161004 & -1.540906 & 1.071570 & 1.695077 \\
\hline 11 & 1 & 10011000 & 0.460146 & -3.814687 & -1.553634 \\
\hline 12 & 6 & 10061003 & -0.167794 & -0.000403 & -3.244302 \\
\hline 13 & 1 & 10011000 & -0.045956 & -1.054630 & -3.512329 \\
\hline 14 & 1 & 10011000 & -1.114260 & 0.369978 & -3.653961 \\
\hline 15 & 1 & 10011000 & 0.654928 & 0.577076 & -3.681779 \\
\hline 16 & 3 & 10031000 & -0.269191 & 2.482221 & -0.002191 \\
\hline 17 & 8 & 10081003 & -1.599362 & 3.361653 & -1.250271 \\
\hline 18 & 8 & 10081003 & -0.049753 & 4.159720 & 1.291922 \\
\hline 19 & 6 & 10061003 & -1.194966 & 3.598848 & -2.558348 \\
\hline 20 & 1 & 10011000 & -0.150267 & 3.246958 & -2.694463 \\
\hline 21 & 1 & 10011000 & -1.854052 & 3.033203 & -3.253978 \\
\hline 22 & 6 & 10061003 & -2.506697 & 4.359742 & -0.916496 \\
\hline 23 & 1 & 10011000 & -3.534788 & 4.017953 & -1.166271 \\
\hline 24 & 1 & 10011000 & -2.459943 & 4.564038 & 0.173650 \\
\hline 25 & 6 & 10061003 & 0.707811 & 5.238968 & 0.842797 \\
\hline 26 & 1 & 10011000 & 0.964691 & 5.100355 & -0.228570 \\
\hline
\end{tabular}




\begin{tabular}{|c|c|c|c|c|c|}
\hline 27 & 1 & 10011000 & 0.101273 & 6.165661 & 0.939450 \\
\hline 28 & 6 & 10061003 & 0.450519 & 3.757936 & 2.530156 \\
\hline 29 & 1 & 10011000 & 0.844838 & 2.725047 & 2.436865 \\
\hline 30 & 1 & 10011000 & -0.368120 & 3.755184 & 3.281081 \\
\hline 31 & 6 & 10061003 & 1.562594 & 4.692271 & 2.961699 \\
\hline 32 & 1 & 10011000 & 1.169513 & 5.448054 & 3.677293 \\
\hline 33 & 1 & 10011000 & 2.411766 & 4.141398 & 3.421803 \\
\hline 34 & 6 & 10061003 & 1.960507 & 5.356166 & 1.683229 \\
\hline 35 & 1 & 10011000 & 2.796363 & 4.797781 & 1.207685 \\
\hline 36 & 1 & 10011000 & 2.255547 & 6.416272 & 1.841012 \\
\hline 37 & 6 & 10061003 & -1.291753 & 5.088508 & -2.827428 \\
\hline 38 & 1 & 10011000 & -0.284306 & 5.558017 & -2.781618 \\
\hline 39 & 1 & 10011000 & -1.749881 & 5.283143 & -3.821775 \\
\hline 40 & 6 & 10061003 & -2.161951 & 5.603432 & -1.711563 \\
\hline 41 & 1 & 10011000 & -3.080183 & 6.092522 & -2.104172 \\
\hline 42 & 1 & 10011000 & -1.596871 & 6.323410 & -1.079824 \\
\hline 43 & 6 & 10061000 & -2.033340 & -3.376896 & -0.834575 \\
\hline 44 & 6 & 10061000 & -3.247786 & -3.244492 & -1.528431 \\
\hline 45 & 6 & 10061000 & -1.824182 & -4.507262 & -0.024918 \\
\hline 46 & 6 & 10061000 & -4.242540 & -4.218498 & -1.400576 \\
\hline 47 & 1 & 10011000 & -3.423714 & -2.390549 & -2.168502 \\
\hline 48 & 6 & 10061000 & -2.823560 & -5.476259 & 0.103717 \\
\hline 49 & 1 & 10011000 & -0.897669 & -4.625688 & 0.523430 \\
\hline 50 & 6 & 10061000 & -4.032153 & -5.331480 & -0.582373 \\
\hline 51 & 1 & 10011000 & -5.178409 & -4.107939 & -1.932644 \\
\hline 52 & 1 & 10011000 & -2.662788 & -6.337142 & 0.739506 \\
\hline 53 & 1 & 10011000 & -4.805759 & -6.081194 & -0.479448 \\
\hline 54 & 6 & 10061000 & -3.168040 & -1.537546 & 1.402159 \\
\hline 55 & 6 & 10061000 & -4.500276 & -1.929480 & 1.186650 \\
\hline 56 & 6 & 10061000 & -2.412697 & -2.186079 & 2.390823 \\
\hline 57 & 6 & 10061000 & -5.063019 & -2.956809 & 1.950036 \\
\hline 58 & 1 & 10011000 & -5.101388 & -1.464654 & 0.417877 \\
\hline 59 & 6 & 10061000 & -2.978550 & -3.212970 & 3.151523 \\
\hline 60 & 1 & 10011000 & -1.381591 & -1.906459 & 2.562751 \\
\hline 61 & 6 & 10061000 & -4.303066 & -3.598496 & 2.931162 \\
\hline 62 & 1 & 10011000 & -6.086229 & -3.262202 & 1.773899 \\
\hline 63 & 1 & 10011000 & -2.388000 & -3.713844 & 3.907536 \\
\hline 64 & 1 & 10011000 & -4.738845 & -4.397389 & 3.516850 \\
\hline 65 & 6 & 10061000 & -3.723475 & 0.646293 & -0.433156 \\
\hline 66 & 6 & 10061000 & -4.641200 & 1.404206 & 0.310616 \\
\hline 67 & 6 & 10061000 & -3.846465 & 0.594734 & -1.830065 \\
\hline 68 & 6 & 10061000 & -5.668058 & 2.099544 & -0.334566 \\
\hline 69 & 1 & 10011000 & -4.564533 & 1.452825 & 1.389355 \\
\hline 70 & 6 & 10061000 & -4.870687 & 1.295805 & -2.473060 \\
\hline 71 & 1 & 10011000 & -3.156967 & 0.016477 & -2.426000 \\
\hline 72 & 6 & 10061000 & -5.781628 & 2.047179 & -1.726203 \\
\hline 73 & 1 & 10011000 & -6.373294 & 2.681852 & 0.243967 \\
\hline 74 & 1 & 10011000 & -4.958778 & 1.255534 & -3.550988 \\
\hline 75 & 1 & 10011000 & -6.574325 & 2.589010 & -2.225485 \\
\hline 76 & 6 & 10061000 & 2.753841 & -2.720463 & -1.014392 \\
\hline 77 & 6 & 10061000 & 3.618994 & -2.795733 & -2.115990 \\
\hline 78 & 6 & 10061000 & 3.078534 & -3.395867 & 0.171899 \\
\hline 79 & 6 & 10061000 & 4.810396 & -3.521543 & -2.022963 \\
\hline 80 & 1 & 10011000 & 3.372402 & -2.288725 & -3.040750 \\
\hline 81 & 6 & 10061000 & 4.272103 & -4.118572 & 0.262022 \\
\hline 82 & 1 & 10011000 & 2.411596 & -3.357601 & 1.024206 \\
\hline 83 & 6 & 10061000 & 5.138058 & -4.180357 & -0.834058 \\
\hline 84 & 1 & 10011000 & 5.481589 & -3.569407 & -2.870501 \\
\hline 85 & 1 & 10011000 & 4.525705 & -4.630756 & 1.180976 \\
\hline 86 & 1 & 10011000 & 6.062870 & -4.737724 & -0.761975 \\
\hline 87 & 6 & 10061000 & 4.289909 & 0.033720 & -0.619206 \\
\hline
\end{tabular}




$\begin{array}{rll}88 & 6 & 10061000 \\ 89 & 6 & 10061000 \\ 90 & 6 & 10061000 \\ 91 & 1 & 10011000 \\ 92 & 6 & 10061000 \\ 93 & 1 & 10011000 \\ 94 & 6 & 10061000 \\ 95 & 1 & 10011000 \\ 96 & 1 & 10011000 \\ 97 & 1 & 10011000 \\ 98 & 6 & 10061000 \\ 99 & 6 & 10061000 \\ 100 & 6 & 10061000 \\ 101 & 6 & 10061000 \\ 102 & 1 & 10011000 \\ 103 & 6 & 10061000 \\ 104 & 1 & 10011000 \\ 105 & 6 & 10061000 \\ 106 & 1 & 10011000 \\ 107 & 1 & 10011000 \\ 108 & 1 & 10011000\end{array}$

$\begin{array}{rr}5.126861 & -0.651789 \\ 4.771547 & 0.357659 \\ 6.428001 & -0.998107 \\ 4.777543 & -0.938048 \\ 6.071892 & 0.006286 \\ 4.137407 & 0.874387 \\ 6.900435 & -0.669617 \\ 7.066635 & -1.531462 \\ 6.436059 & 0.255392 \\ 7.905784 & -0.944270 \\ 2.464815 & 0.328742 \\ 3.234762 & 1.152250 \\ 1.572043 & -0.591590 \\ 3.114730 & 1.054986 \\ 3.922540 & 1.872730 \\ 1.454746 & -0.686311 \\ 0.956891 & -1.229000 \\ 2.224648 & 0.136680 \\ 3.708210 & 1.694940 \\ 0.763065 & -1.394874 \\ 2.130123 & 0.064107\end{array}$

2

A

16.3967

5.2312

0.0008

0.3182
0.278193

$-1.896409$

$-0.098320$

1. 260146

$-2.270451$

$-2.605726$

$-1.371346$

0.593762

$-3.258675$

$-1.663069$

1. 622602

2. 458216

2.194583

3. 847375

2.033805

3. 584196

1. 575811

4. 410214

4.487230

4.020992

5.485864

Frequencies - -

Red. masses --
Frc consts - -

5.3495

IR Inten

0.1103

$\mathrm{HF}=-3912.8445276$

Optimized geometry, three lower frequencies and SCF energy for IIb

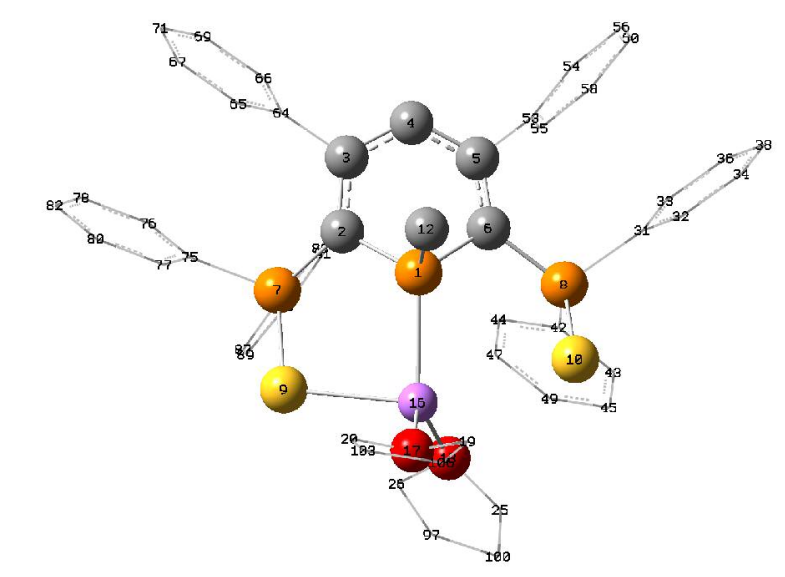

\begin{tabular}{|c|c|c|c|c|c|}
\hline \multirow{2}{*}{$\begin{array}{l}\text { Center } \\
\text { Number }\end{array}$} & \multirow{2}{*}{$\begin{array}{l}\text { Atomic } \\
\text { Number }\end{array}$} & \multirow{2}{*}{$\begin{array}{r}\text { Atomic } \\
\text { Type }\end{array}$} & \multicolumn{3}{|c|}{ Coordinates (Angstroms) } \\
\hline & & & $\mathrm{x}$ & Y & Z \\
\hline . & 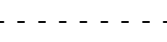 & --- & $-\ldots-\ldots$ & $-\ldots-\ldots$ & $-\ldots-\ldots$ \\
\hline 1 & 15 & 0 & -0.128720 & 0.337685 & -1.390152 \\
\hline 2 & 6 & 0 & -1.539784 & -0.538517 & -0.707086 \\
\hline 3 & 6 & 0 & -1.532712 & -1.953294 & -0.831048 \\
\hline 4 & 6 & 0 & -0.344860 & -2.670823 & -0.982604 \\
\hline 5 & 6 & 0 & 0.954749 & -2.127997 & -0.776499 \\
\hline 6 & 6 & 0 & 1.221360 & -0.765887 & -0.854405 \\
\hline 7 & 15 & 0 & -2.606074 & 0.434070 & 0.335080 \\
\hline
\end{tabular}




\begin{tabular}{|c|c|c|c|c|c|}
\hline 8 & 15 & 0 & 2.742265 & 0.144864 & -0.509605 \\
\hline 9 & 16 & 0 & -2.428142 & 2.427507 & 0.098773 \\
\hline 10 & 16 & 0 & 2.903335 & 1.859380 & -1.523597 \\
\hline 11 & 1 & 0 & -0.416066 & -3.752464 & -1.100000 \\
\hline 12 & 6 & 0 & -0.210760 & -0.039981 & -3.204583 \\
\hline 13 & 1 & 0 & -0.290213 & -1.115438 & -3.391706 \\
\hline 14 & 1 & 0 & -1.082671 & 0.466166 & -3.635420 \\
\hline 15 & 1 & 0 & 0.700930 & 0.343940 & -3.675743 \\
\hline 16 & 3 & 0 & 0.047955 & 2.693776 & -0.648700 \\
\hline 17 & 8 & 0 & -0.180308 & 4.024927 & -2.142018 \\
\hline 18 & 8 & 0 & 0.868096 & 3.270401 & 1.092874 \\
\hline 19 & 6 & 0 & 0.755969 & 4.040103 & -3.168741 \\
\hline 20 & 6 & 0 & -1.438808 & 4.041885 & -2.739660 \\
\hline 21 & 1 & 0 & 1.690350 & 4.528661 & -2.819645 \\
\hline 22 & 1 & 0 & 0.985384 & 2.995166 & -3.473450 \\
\hline 23 & 1 & 0 & -1.835413 & 3.003898 & -2.790138 \\
\hline 24 & 1 & 0 & -2.129514 & 4.657212 & -2.124700 \\
\hline 25 & 6 & 0 & 1.904789 & 4.198164 & 1.116384 \\
\hline 26 & 6 & 0 & 0.029179 & 3.557784 & 2.163779 \\
\hline 27 & 1 & 0 & 1.670657 & 5.024418 & 0.409593 \\
\hline 28 & 1 & 0 & 2.846984 & 3.713231 & 0.788495 \\
\hline 29 & 1 & 0 & -0.774073 & 4.249169 & 1.826155 \\
\hline 30 & 1 & 0 & -0.435408 & 2.620118 & 2.536081 \\
\hline 31 & 6 & 0 & 4.140658 & -0.913054 & -0.950014 \\
\hline 32 & 6 & 0 & 4.965109 & -1.498174 & 0.026632 \\
\hline 33 & 6 & 0 & 4.401039 & -1.162225 & -2.305966 \\
\hline 34 & 6 & 0 & 6.036639 & -2.312165 & -0.352927 \\
\hline 35 & 1 & 0 & 4.774934 & -1.351963 & 1.079956 \\
\hline 36 & 6 & 0 & 5.470994 & -1.979651 & -2.681409 \\
\hline 37 & 1 & 0 & 3.769897 & -0.730103 & -3.072400 \\
\hline 38 & 6 & 0 & 6.289715 & -2.552866 & -1.705479 \\
\hline 39 & 1 & 0 & 6.663992 & -2.766438 & 0.402960 \\
\hline 40 & 1 & 0 & 5.663517 & -2.170812 & -3.729081 \\
\hline 41 & 1 & 0 & 7.115803 & -3.188366 & -1.996818 \\
\hline 42 & 6 & 0 & 2.873170 & 0.532220 & 1.250700 \\
\hline 43 & 6 & 0 & 4.006282 & 1.203120 & 1.735659 \\
\hline 44 & 6 & 0 & 1.826673 & 0.225469 & 2.131089 \\
\hline 45 & 6 & 0 & 4.102447 & 1.534739 & 3.090110 \\
\hline 46 & 1 & 0 & 4.813935 & 1.467716 & 1.064897 \\
\hline 47 & 6 & 0 & 1.923424 & 0.559628 & 3.484740 \\
\hline 48 & 1 & 0 & 0.934592 & -0.261945 & 1.772122 \\
\hline 49 & 6 & 0 & 3.062627 & 1.210362 & 3.965089 \\
\hline 50 & 1 & 0 & 4.979644 & 2.049511 & 3.459792 \\
\hline 51 & 1 & 0 & 1.111990 & 0.323065 & 4.160012 \\
\hline 52 & 1 & 0 & 3.134476 & 1.473321 & 5.012394 \\
\hline 53 & 6 & 0 & 1.995585 & -3.099029 & -0.371134 \\
\hline 54 & 6 & 0 & 2.618409 & -3.907086 & -1.334274 \\
\hline 55 & 6 & 0 & 2.315917 & -3.262903 & 0.985162 \\
\hline 56 & 6 & 0 & 3.576127 & -4.848748 & -0.945796 \\
\hline 57 & 1 & 0 & 2.368585 & -3.799674 & -2.382639 \\
\hline 58 & 6 & 0 & 3.272407 & -4.207240 & 1.370097 \\
\hline 59 & 1 & 0 & 1.823108 & -2.665268 & 1.741015 \\
\hline 60 & 6 & 0 & 3.904484 & -4.997328 & 0.405109 \\
\hline 61 & 1 & 0 & 4.065197 & -5.461516 & -1.691762 \\
\hline 62 & 1 & 0 & 3.523374 & -4.326654 & 2.415955 \\
\hline 63 & 1 & 0 & 4.647818 & -5.724817 & 0.704046 \\
\hline 64 & 6 & 0 & -2.797063 & -2.727633 & -0.805953 \\
\hline 65 & 6 & 0 & -3.734353 & -2.570664 & -1.838718 \\
\hline 66 & 6 & 0 & -3.047871 & -3.650670 & 0.222054 \\
\hline 67 & 6 & 0 & -4.919776 & -3.311726 & -1.830246 \\
\hline 68 & 1 & 0 & -3.547523 & -1.872329 & -2.645419 \\
\hline
\end{tabular}




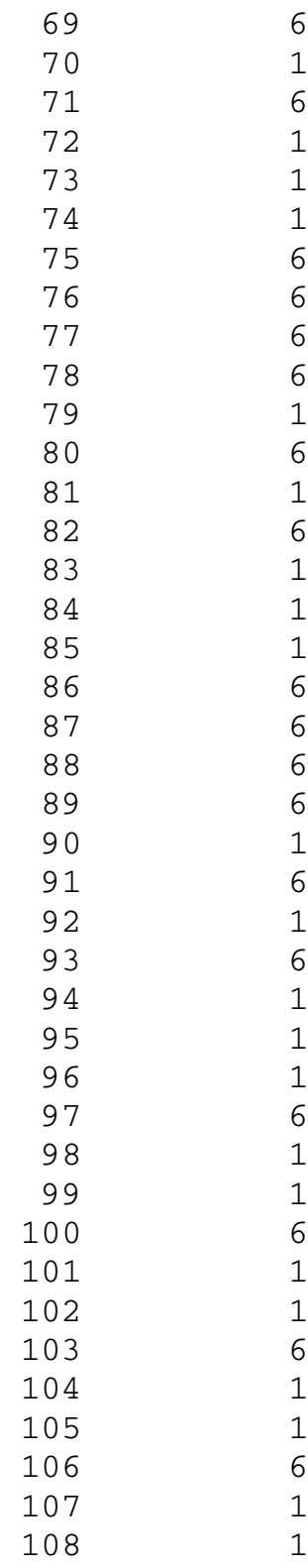

$\begin{array}{rr}-4.236844 & -4.386360 \\ -2.328849 & -3.793021 \\ -5.172722 & -4.216045 \\ -5.644731 & -3.180490 \\ -4.432479 & -5.089034 \\ -6.093425 & -4.784736 \\ -4.328285 & -0.008408 \\ -5.050389 & -0.841390 \\ -4.953178 & 0.479953 \\ -6.379344 & -1.171414 \\ -4.590849 & -1.253023 \\ -6.281459 & 0.145467 \\ -4.409242 & 1.111501 \\ -6.994719 & -0.678462 \\ -6.929116 & -1.818319 \\ -6.756547 & 0.521953 \\ -8.021692 & -0.939997 \\ -2.334414 & 0.080222 \\ -2.903272 & 0.918785 \\ -1.578390 & -1.029302 \\ -2.702632 & 0.662371 \\ -3.504157 & 1.770019 \\ -1.382174 & -1.284922 \\ -1.125921 & -1.693573 \\ -1.940983 & -0.438137 \\ -3.139758 & 1.315924 \\ -0.790662 & -2.136905 \\ -1.785007 & -0.635434 \\ 0.849960 & 4.209618 \\ 0.282088 & 5.026570 \\ 1.152113 & 3.453997 \\ 2.057144 & 4.735394 \\ 2.992066 & 4.357702 \\ 2.067209 & 5.847433 \\ -1.318134 & 4.624047 \\ -1.733706 & 3.917788 \\ -1.844857 & 5.600711 \\ 0.161969 & 4.798161 \\ 0.504162 & 4.382741 \\ 0.434341 & 5.874627\end{array}$

2

18.6349

4.6896

0.0010

0.3024
0.229562

1. 019057

$-0.794857$

$-2.622944$

1. 029034

$-0.786564$

0.009179

0.881688

$-1.148270$

0.599845

1.769268

$-1.427686$

$-1.839416$

$-0.553462$

1. 271057

$-2.324208$

$-0.772771$

2.092605

3.064481

2.503693

4.423856

2. 771790

3. 863597

1. 785024

4.823447

5.167547

4.172897

5.876050

3. 255410

3.751733

4. 012809

2. 528991

2.998148

2. 526179

$-4.139148$

$-4.891444$

$-4.214905$

$-4.333740$

$-5.306644$

$-4.265152$

3

A

19.7701

4.9079

0.0011

0.4894

$\mathrm{HF}=-3912.8420524$ 


\section{Complete Reference (16)}

Frisch, M. J.; Trucks, G. W.; Schlegel, H. B.; Scuseria, G. E.; Robb, M. A.; Cheeseman, J. R.; Jr., J. A. M.; Vreven, T.; Kudin, K. N.; Burant, J. C.; Millam, J. M.; Iyengar, S. S.; Tomasi, J.; Barone, V.; Mennucci, B.; Cossi, M.; Scalmani, G.; Rega, N.; Petersson, G. A.; Nakatsuji, H.; Hada, M.; Ehara, M.; Toyota, K.; Fukuda, R.; Hasegawa, J.; Ishida, M.; Nakajima, T.; Honda, Y.; Kitao, O.; Nakai, H.; Klene, M.; Li, X.; Knox, J. E.; Hratchian, H. P.; Cross, J. B.; Adamo, C.; Jaramillo, J.; Gomperts, R.; Stratmann, R. E.; Yazyev, O.; Austin, A. J.; Cammi, R.; Pomelli, C.; Ochterski, J. W.; Ayala, P. Y.; Morokuma, K.; Voth, G. A.; Salvador, P.; Dannenberg, J. J.; Zakrzewski, V. G.; Dapprich, S.; Daniels, A. D.; Strain, M. C.; Farkas, O.; Malick, D. K.; Rabuck, A. D.; Raghavachari, K.; Foresman, J. B.; Ortiz, J. V.; Cui, Q.; Baboul, A. G.; Clifford, S.; Cioslowski, J.; Stefanov, B. B.; Liu, G.; Liashenko, A.; Piskorz, P.; Komaromi, I.; Martin, R. L.; Fox, D. J.; Keith, T.; Al-Laham, M. A.; Peng, C. Y.; Nanayakkara, A.; Challacombe, M.; Gill, P. M. W.; Johnson, B.; Chen, W.; Wong, M. W.; Gonzalez, C.; Pople, J. A. In; Revision C.02 ed.; Gaussian, Inc.: Wallingford CT, 2004. 